\title{
Plasticité et endommagement du sel gemme
}

\section{THOREL}

\section{GHOREYCHI}

Groupement pour l'étude des structures souterraines de stockage G.3S - LMS (URA 317),

École polytechnique, 91128 Palaiseau Cedex
L'apparition de déformations permanentes, instantanées ou différées, sous le moindre chargement déviatorique, caractérise la rhéologie du sel gemme. Sous certaines conditions de chargement, l'endommagement apparaît, pouvant nuire à la sûreté d'ouvrages souterrains de stockage, notamment par accroissement de la perméabilité.

Des essais de laboratoire de compression et d'extension sous chargement axisymétrique ont été réalisés afin de caractériser le comportement du sel gemme au cours de son endommagement. La mesure des déformations volumiques en continu permet d'identifier l'initiation de Y'endommagement, liée à l'apparition de dilatance. Un critère d'endommagement ouvert dans l'espace des contraintes est ainsi établi. Ce critère tient compte de la précocité de l'endommagement en extension par rapport à la compression.

Sur la base des résultats expérimentaux, un modèle est proposé afin de prendre en compte la plasticité et l'endommagement du sel gemme. Suivant un formalisme de plasticité non standard, ce modèle est généralisé à tout l'espace des contraintes. Le paramètre d'écrouissage est la distorsion plastique. Les choix de la surface de charge et du potentiel plastique sont reliés à l'analyse de l'évolution de l'angle de dilatance. Enfin, le modèle est appliqué au cas du calcul de structures souterraines simplifiées, faisant apparaitre les risques d'endommagement en milieu salifère.

\section{Plasticity and damage of rocksalt}

Instantaneous or delayed permanent strains generated under small deviatoric stress are characteristic of rocksalt rheology. Development of damage for particular stress state, often implies permeability increase, which compromise the safety of underground storage cavities.

To study damage evolution in rocksalt, under different stress paths, compression and extension axisymmetric triaxial tests are performed in the laboratory. As damage is linked to

development of micro viods and microcracks, and identified to irreversible dilatancy, volume change measurements are carried out during the tests. The stress state corresponding to the dilatancy onset, allows the identification of a damage initiation criterion. This criterion is proposed as an open surface in the stress space, including the effect of the damage precocity under extension stress state.

Before and during damage evolution, rocksalt exhibits plastic strains. An elastic-plastic model, 3D generalised, is proposed. It considers isotropic hardening and non-associtated flow rule with plastic distortion as a hardening parameter. Model parameters are fitted using experimental data for the evolution of the dilatancy angle. A numerical application to spherical and cylindrical cavities is given, showing the damage risk assessment. 


\section{NOTATIONS}

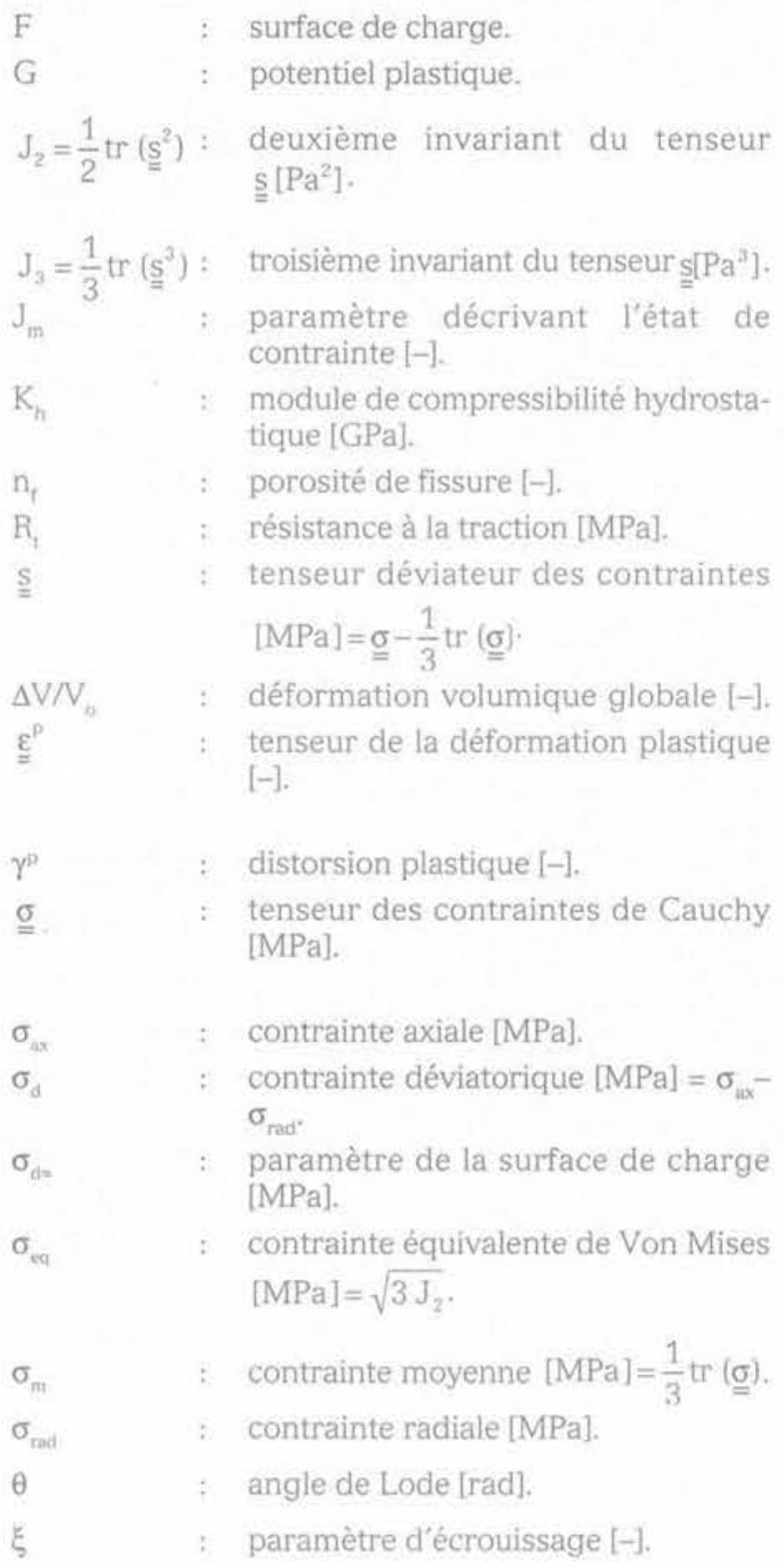

\section{Introduction}

De nombreuses cavités de stockage souterrain ont été réalisées dans le sel gemme, en raison notamment de ses propriétés de faible perméabilité et de faible porosité. Cependant, la réalisation et l'exploitation des cavités peut générer un endommagement dans le massif ce qui, par augmentation notable de la perméabilité, peut engendrer des problèmes de sûreté à plus ou moins long terme.

Le comportement du sel gemme endommagé est étudié depuis peu tant sur le plan expérimental (Hunsche, 1984, 1993; Hunsche et al., 1990) que sur le plan théorique (Aubertin et al, 1991, 1993; Cristescu, 1989, 1993 ; Pouya, 1991; Thorel et Ghoreychi, 1993). Le comportement à court terme revêt une importance particulière lors du creusement des ouvrages. Sous l'action de contraintes déviatoriques, les mécanismes de glissement dans les grains de chlorure de sodium s'activent et, en raison d'une imparfaite accommodation des déformations à l'interface des grains, génèrent des concentrations de contraintes voire des microfissures (Stokes, 1966). Le sel gemme développe alors des déformations élastoplastiques, accompagnées d'un endommagement selon des conditions qui seront précisées plus loin.

Sur la base de résultats expérimentaux de laboratoire (essais de compression et d'extension), un modèle d'endommagement fondé sur le formalisme d'élastoplasticité non standard est proposé. Sa mise en œuvre à travers un exemple de calcul de structure simplifiée permet une discussion quant aux conséquences de l'endommagement en milieu salifère.

\section{2}

\section{Identification de l'endommagement}

L'endommagement est la création et le développement de microfissures ou de microvides au sein de la matière (Lemaitre et al., 1978). Quelques facteurs physiques et mécaniques (pression, température, vitesse de chargement) régissent les effets de « ductilité » et de « fragilité " dans le comportement du sel gemme, qui appartient à la classe des matériaux semi-fragile à ductile. Parmi les diverses méthodes d'identification de l'endommagement, nous avons retenu la mesure de la déformation volumique. Cette mesure peut en effet donner directement des informations sur la dilatance du matériau causée par l'apparition de microfissures ou de microvides, et donc traduire un certain état d'endommagement. En veillant à prendre quelques précautions, notamment quant au maintien d'une température constante pendant l'essai, la mesure de la déformation volumique globale présente plusieurs avantages : elle est continue au cours d'un essai sous chargement déviatorique, même lorsque l'éprouvette est fortement déformée ; sa mise en œuvre est aisée (ce qui n'est pas le cas des jauges collées).

Le choix de cette méthode dans le cas du sel gemme est d'autant plus justifié que les mécanismes de déformation du monocristal de $\mathrm{NaCl}$ (mouvements de dislocations, diffusion, dissolution-recristallisation) génèrent des déformations volumiques très faibles, voire négligeables (de l'ordre de 10-7 (Davidge et al, 1964) pour le mouvement des dislocations). Sur le polycristal de sel, une dilatance supérieure de plusieurs ordres de grandeurs à celle du monocristal, pourra être reliée à un endommagement, attribué à une microfissuration et aboutissant à une augmentation de la perméabilité (Spiers et al., 1989 ; Peach, 1993). Il n'est donc pas surprenant que la dilatance du sel gemme montre également une bonne corrélation avec d'autres indicateurs d'endommagement, tels que l'émission acoustique (Hunsche, 1984).

\section{3}

\section{Résultats expérimentaux}

Des essais triaxiaux axisymétriques de compression et d'extension (sous chargement compressif) sont réalisés avec mesure de la déformation volumique. Les trajets de chargement sont représentés sur la figure 1, en utilisant la convention des compressions négatives. Le sel gemme testé (d'âge Stampien), provient des Mines De Potasse d'Alsace (MDPA). Extrait du banc S1, il s'agit d'un sel relativement pur (au moins $90 \%$ de $\mathrm{NaCl}$ ) dont les grains sont de dimension plurimillimétrique à centimétrique. 

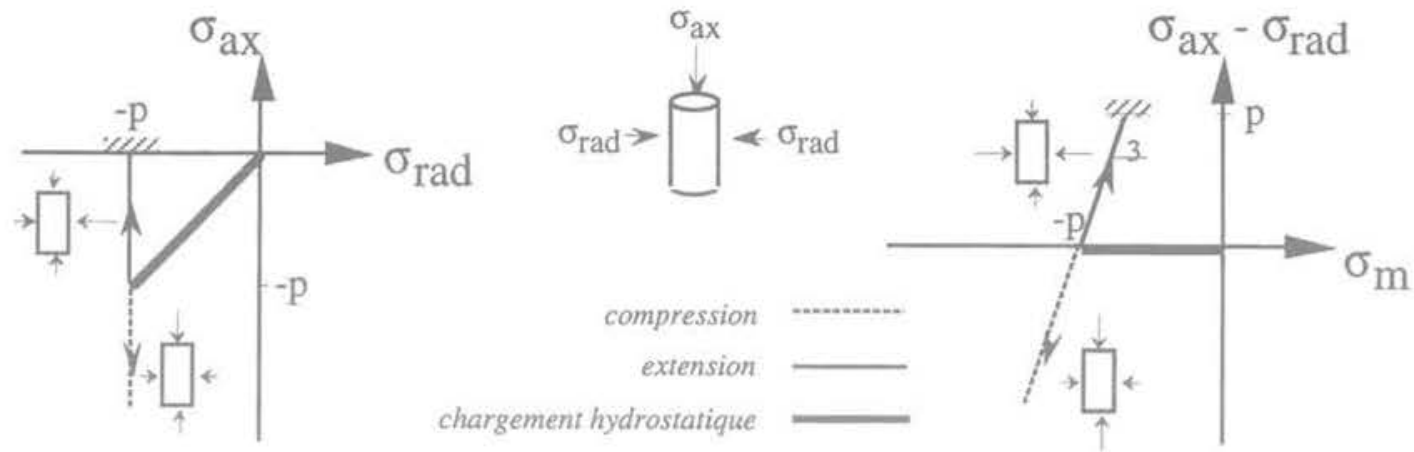

fig.

Trajets de chargement de compression axiale et d'extension axiale.

Compression and extension stress paths.

\section{Dispositif expérimental et programme d'essai}

Le dispositif expérimental se compose essentiellement d'une presse hydraulique MTS asservie électroniquement, d'une cellule triaxiale de capacité maximale $70 \mathrm{MPa}$, d'un contrôleur numérique pression-volume GDS (pression maximale $64 \mathrm{MPa}$ ) connecté hydrauliquement à la cellule, permettant la mesure des variations du volume d'huile contenu dans la cellule et le contrôle de la pression de confinement. La mesure de la déformation axiale, effectuée à l'extérieur de la cellule mais à proximité de l'éprouvette, est déduite du déplacement du piston. Dans le cas de l'essai unaxial, la déformation volumique est déduite des mesures extensométriques de déformation axiale et circonférentielle, obtenues par des capteurs MTS fixés sur l'éprouvette.

Après le chargement hydrostatique, un chargement déviatorique est appliqué soit en augmentant la contrainte axiale (compression), soit en la relâchant (extension), ou en alternant des phases de compression et d'extension. Les éprouvettes ont un diamètre compris entre $50 \mathrm{~mm}$ et $60 \mathrm{~mm}$ et un élancement égal à 2 . Les essais couvrent une gamme de pressions de confinement comprises entre 0 et $60 \mathrm{MPa}$ en compression et 20 à $60 \mathrm{MPa}$ en extension. La mise sous confinement se fait à une vitesse de $10 \mathrm{kPa} . \mathrm{s}^{-1}$ et nécessite une régulation de la contrainte radiale (pression de fluide) par rapport à la contrainte axiale (force appliquée par la presse). Cette régulation est assurée par un pilotage numérique. Le chargement déviatorique est contrôlé en déformation axiale à une vitesse de l'ordre de $10^{-5} \mathrm{~S}^{-1}$.

\section{2}

\section{Résultats typiques}

Les résultats sont interprétés en supposant que l'état de déformation actuel de l'éprouvette se déduit de l'état initial par une transformation cylindrique. Cette hypothèse est plus ou moins vérifiée grâce aux précautions prises pour réduire le frottement entre le piston et l'éprouvette, à l'aide d'un lubrifiant. Les directions principales du tenseur des contraintes et du tenseur des déformations demeurent constantes. Les effets de bord sont ainsi négligés.

\subsection{1}

\section{Chargement hydrostatique}

Au cours du chargement hydrostatique, un cycle de décompression (Fig. 2) permet d'accéder au module de compressibilité hydrostatique $\left(\mathrm{K}_{\mathrm{h}}\right.$ moyen $\left.=7,7 \mathrm{GPa}\right)$ et à la porosité de fissures ( $n$, inférieure à $0,5 \%$ ). La déformation volumique est dans ce cas déterminée à partir de la déformation axiale, en supposant que les déformations sont petites et isotropes.

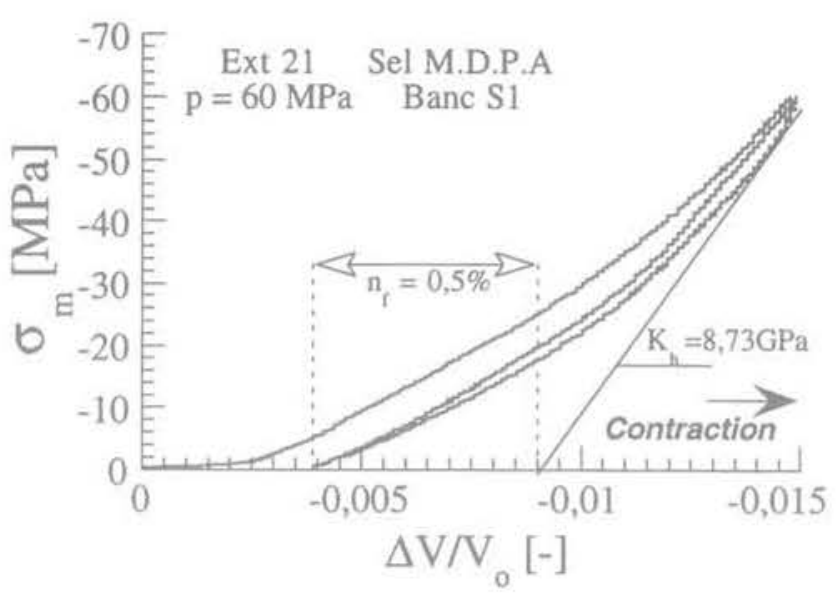

FG.2 Chargement hydrostatique d'une éprouvette. Hydrostatic loading.

Le contrainte moyenne est définie par:

$$
\sigma_{\mathrm{m}}=\frac{1}{3} \mathrm{tr} \underline{\underline{\sigma}}
$$

\section{9}

\section{Chargement déviatorique}

Le comportement du sel gemme sous chargement déviatorique est élastoplastique avec écrouissage (Fig. 3 et 4), essentiellement isotrope. On observe un effet Bauschinger en cas de chargement alterné (Fig. 5).

La répartition des contraintes principales, différente en compression et en extension, engendre une fissuration diffuse pour laquelle les plans de fissuration sont 
respectivement subaxiaux et perpendiculaires à la génératrice de l'éprouvette cylindrique. L'intensification de la contrainte moyenne en compression et son relâchement en extension, influent sur la déformation volumique $\Delta \mathrm{V} / \mathrm{V}_{0}$ (respectivement contraction et dilatation): on n'observe pas de contractance en extension, alors que le chargement en compression peut générer une dilatance, après une phase de contractance (Fig. 3). Le calcul des contraintes de Cauchy se fait dans la configuration actuelle, en supposant que l'éprouvette conserve une forme cylindrique au cours de la transformation, c'est-à-dire en négligeant les effets de bord. Les variations de section sont donc prises en compte dans le calcul de la contrainte axiale.

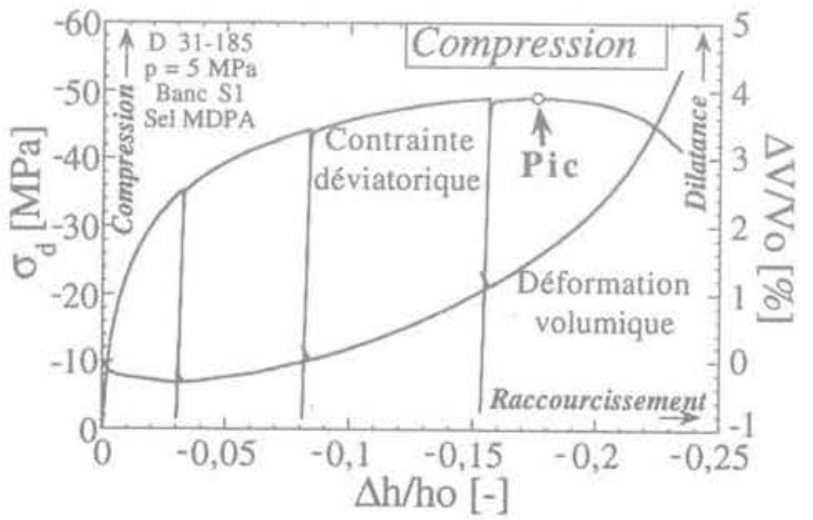

FG. 3 Essai typique de compression. Typical result of a compression test.
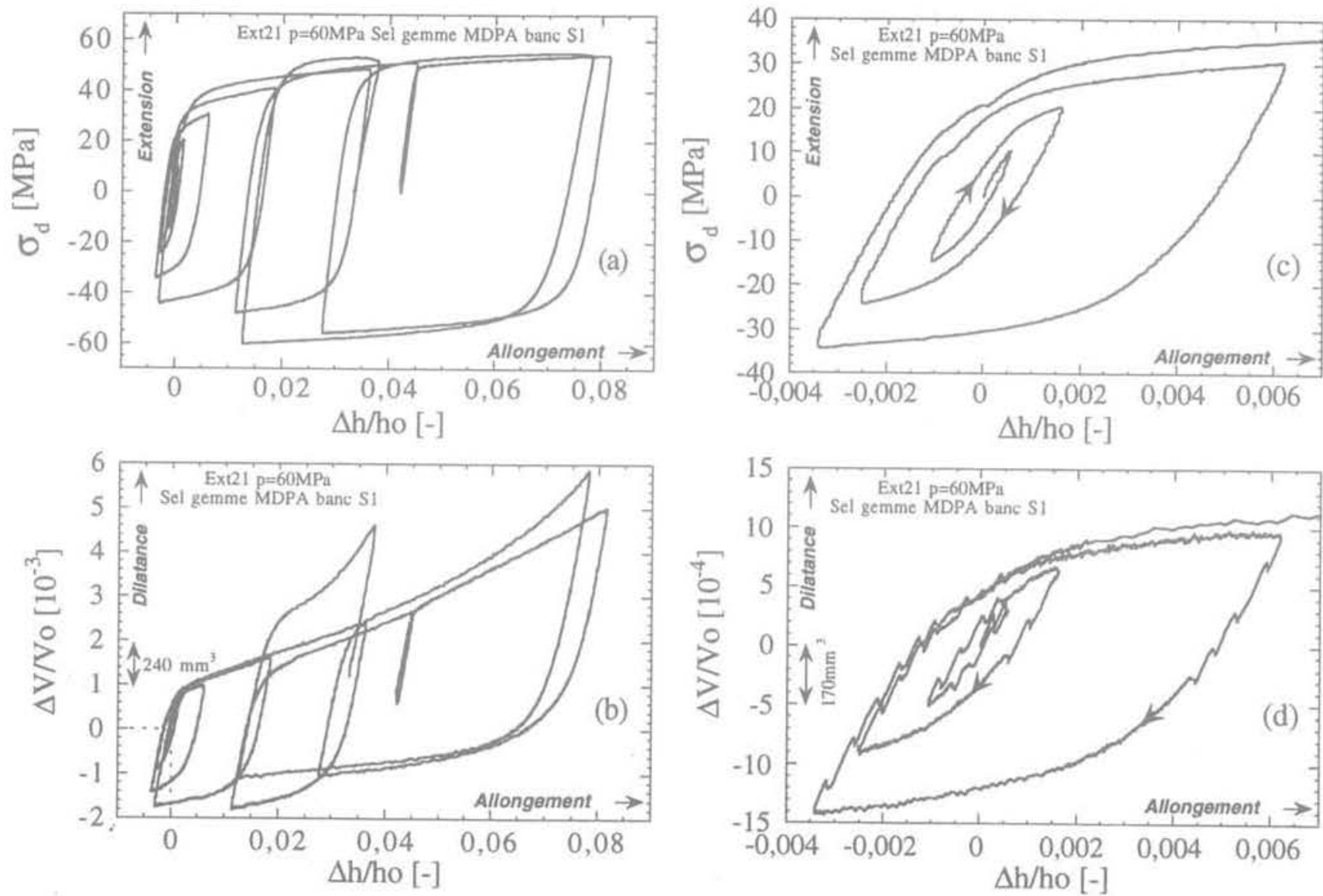

En compression, la contrainte déviatorique $\sigma_{\mathrm{d}}$ est négative et peut être non monotone : le pic de contrainte qui définit conventionnellement la rupture macroscopique, est suivi d'une phase de radoucissement. Cette dernière n'est atteinte que pour des pressions de confinement inférieures à $12 \mathrm{MPa}$ (contrainte moyenne $\left|\sigma_{m}\right|>30 \mathrm{MPa}$ ) (Thorel et Ghoreychi, 1993). En extension (Fig. 4), $\sigma_{d}$ est positive et toujours croissante au cours du chargement ; il n'y a pas de radoucissement. Pour l'observer il faudrait pénétrer dans le domaine de la traction.

Les résultats d'un essai avec chargement alterné en compression et extension sont présentés sur la figure 5.

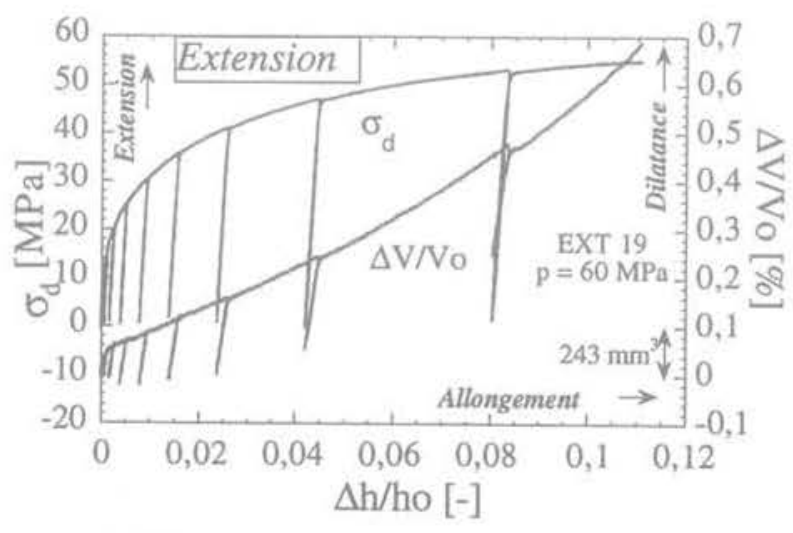

FG.4 Exemple d'essai d'extension. An example of extension test.

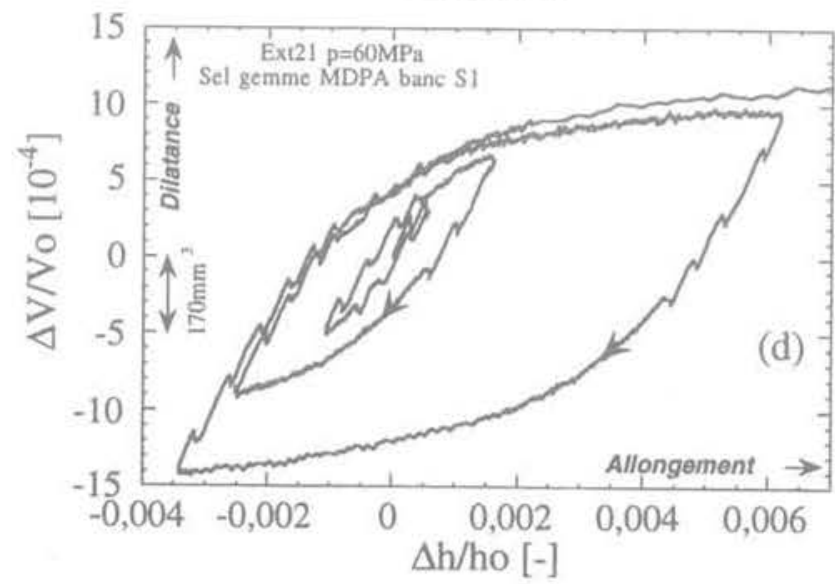


Les figures ( $5 a$ ) et (5b) montrent respectivement l'évolution de la contrainte déviatorique $\sigma_{f}$ et de la déformation volumique $\Delta V / V$ en fonction dé la déformation axiale $\Delta h / h$. Les figures $(5 c)$ et $(5 d)$ sont des agrandissements des courbes au début de l'essai. Le point de départ des courbes correspond exactement à l'origine du chargement. Tant que la contrainte déviatorique est inférieure (en valeur absolue) à $20 \mathrm{MPa}$ environ, le comportement en compression et en extension demeure grossièrement symétrique, par rapport à l'origine. Audelà, la déformation axiale en extension croît pour une faible augmentation du déviateur, ce qui conduit à déplacer peu à peu les cycles dans le domaine des déformations permanentes en extension.

Les cycles de décharge permettent de déterminer les paramètres élastiques isotropes (module d'Young $\mathrm{E}=$ $14,8 \mathrm{GPa}$, coefficient de Poisson $v=0,17$, module de compressibilité $\mathrm{K} \approx 7.5 \mathrm{GPa}$, module de cisaillement $\mathrm{G}=6,3 \mathrm{GPa}$ ). La mesure locale des déformations (avec un extensiomètre à griffes et un collier extensométrique placés directement sur l'éprouvette) en compression simple donne, par rapport à la mesure globale, des modules d'Young E et de cisaillement G supérieurs (près du double de leur valeur déduite des mesures globales), le coefficient de Poisson v et le module de compressibilité K étant peu modifiés. L'influence du type de mesure de déformation (jauge ou extensomètre, locale ou globale) a déjà été étudiée sur le sel gemme et la potasse (Laitai et al., 1988) et les résultats obtenus la corroborent. La vitesse de chargement comprise entre $10^{-7}$ et $10^{-3} \mathrm{~s}^{-1}$ n'a pas d'influence sur les paramètres élastiques.

A l'échelle de l'éprouvette décimétrique, le sel gemme est initialement isotrope (confirmation par mesures acoustiques). Une faible anisotropie induite par la fissuration apparait au cours de chargement déviatorique. Cependant, les variations des paramètres élastiques étant noyées dans la dispersion naturelle des résultats, nous considérons que les paramètres élastiques sont constants.

\section{3}

\section{Limite de linéarité}

L'initiation de l'endommagement est souvent repérée, lors d'un essai de compression, par le seuil de contractance-dilatance, dénommé également seuil de foisonnement ou seuil caractéristique (Habib, 1953 ; Luong, 1981) défini, comme le minimum de la déformation volumique totale. Cette définition devint inopérante en extension. Afin de définir un critère valable dans les deux cas de figure, nous proposons la limite de linéarité de la courbe $\left\{\sigma_{m} ; \Delta \mathrm{V} / \mathrm{V}_{\mathrm{o}}\right\}$.
Nous montrons, en compression tout comme en extension, que les déformations volumiques demeurent réversibles tant que la limite de linéarité n'est pas dépassée (Fig. 6), alors que des déformations déviatoriques irréversibles apparaissent dès l'application d'un déviateur des contraintes. Les figures $6 a$ et $6 \mathrm{~b}$ se réfèrent au même essai de compression simple, pour lequel sont comparées les décharges (1) et (2). La figure $6 c$ se réfère au même essai d'extension que celui de la figure 4.

Le report des états de contrainte relatifs aux limites de linéarité dans le plan déviatorique (Fig. 7) permet de constater que l'endommagement apparaît en extension pour un déviateur plus faible en valeur absolue qu'en compression.

La prècocité de l'endommagement en extension apparaît aussi sur l'essai à chargement alterné (Fig. 5). Dès que la limite de linéarité en extension est franchie, les déformations axiales irréversibles sont plus importantes au cours d'un trajet de chargement en extension qu'au cours du trajet inverse en compression, ce qui conduit au décalage progressif des $\alpha$ boucles $)$ vers les déformations en extension.

L'état de contrainte correspondant à la limite de linéarité, et celui du pic de contrainte en compression permettent de définir des domaines respectivement d'initiation de l'endommagement et de rupture (Fig. 7). Les critères sont d'allure parabolique dans le plan $l \sigma_{m}$; $\left.\sigma_{d}\right)$ (pour les pressions de confinement utilisées, la fermeture du domaine n'est pas observée), ce qui permet de prendre en compte l'influence croissante de la contrainte moyenne dans le domaine des faibles pressions de confinement.

Sur la figure 8, est comparée la courbe d'initiation de l'endommagement avec des données rassemblées par Van Sambeek et al. (1993) sur les conditions d'apparition de dilatance du sel gemme au cours d'essais de fluage. On constate que la surface identifiée à court terme, sépare presque parfaitement les états de contraintes donnant lieu à long terme à une dilatance, de ceux ne manifestant pas de dilatance. Il en ressort que le comportement à long terme du sel gemme peut être modifié suivant que le matériau est endommagé à court terme ou non.

Les résultats expérimentaux permettent de conclure que, pour prendre en compte le comportement observé lors des trajets de chargement de compression et d'extension, il faut distinguer ces trajets, ce qui implique l'usage d'un paramètre dépendant du troisième invariant du tenseur des contraintes (une fonction de l'angle de Lode $\theta$ par exemple). Nous utilisons une variable adimensionnelle $J_{m}$ fonction du second et du troisième invariant du tenseur déviateur des

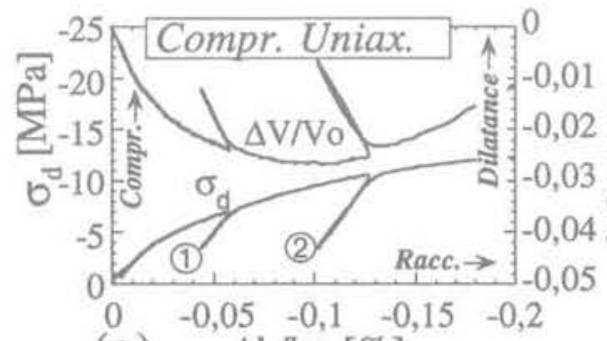

(a) $\Delta \mathrm{h} / \mathrm{ho}[\%]$

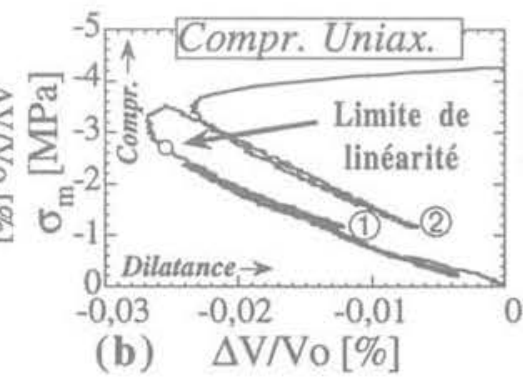

(b) $\Delta \mathrm{V} / \mathrm{Vo}[\%]$

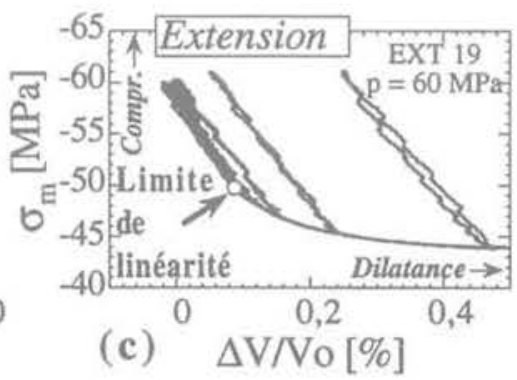

FiG. 6 Limite de linéarité en compression uniaxiale (a et b) et en extension (c). Linearity limit under compression and extension loading. 


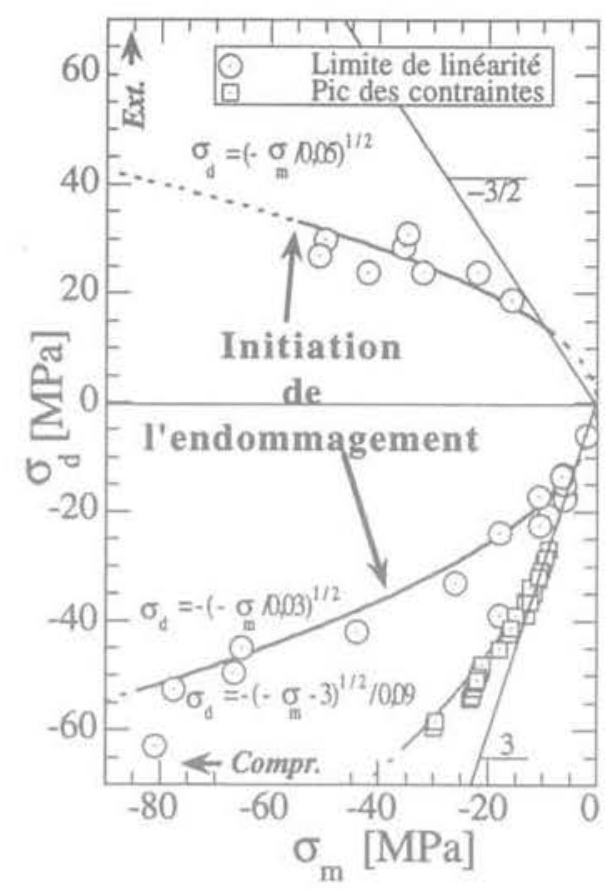

คG.7 Rupture et initiation de l'endommagement dans le plan des contraintes.

Failure and damage onset lines in the stress plane.

contraintes. Ce choix a déjà été adopté par d'autres auteurs (Hunsche, 1984) :

$$
J_{m}=\frac{3 J_{3} \sqrt{3}}{2 J_{2}^{3 / 2}}=\sin 3 \theta
$$

Pour des essais triaxiaux axisymétriques, si l'on fait l'hypothèse qu'en tout point de l'éprouvette cylindrique, au maximum deux des contraintes principales sont différentes, ce paramètre ne peut prendre que deux valeurs distinctes : $J_{m}=1$ en extension et $J_{m}=-1$ en compression. Cela signifie que l'étude d'autres

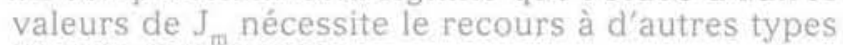
d'essais de laboratoire.

\section{4}

\section{Mise au point d'un modèle rhéologique}

En s'appuyant sur les résultats expérimentaux, le modèle proposé a pour objectif de rendre compte tant qualitativement que quantitativement du comportement du sel gemme dans la phase durcissante, en petites déformations. L'anisotropie induite par la fissuration n'a volontairement pas fait l'objet de la modélisation. En effet, ce phénomène lié à l'apparition de forts gradients de déformation et au développement de déformations localisées se manifeste essentiellement dans la phase post-pic. On s'attache à limiter le nombre des variables, tout en reproduisant au mieux le comportement.

On retient une approche en élastoplasticité avec écrouissage. Un seul mécanisme d'écoulement est pris en compte. L'hypothèse d'un écoulement non associé est formulée a priori. Ce choix est motivé par la nécessité d'un calcul précis de la déformation volumique du sel gemme, de laquelle dépend fortement la perméabi-

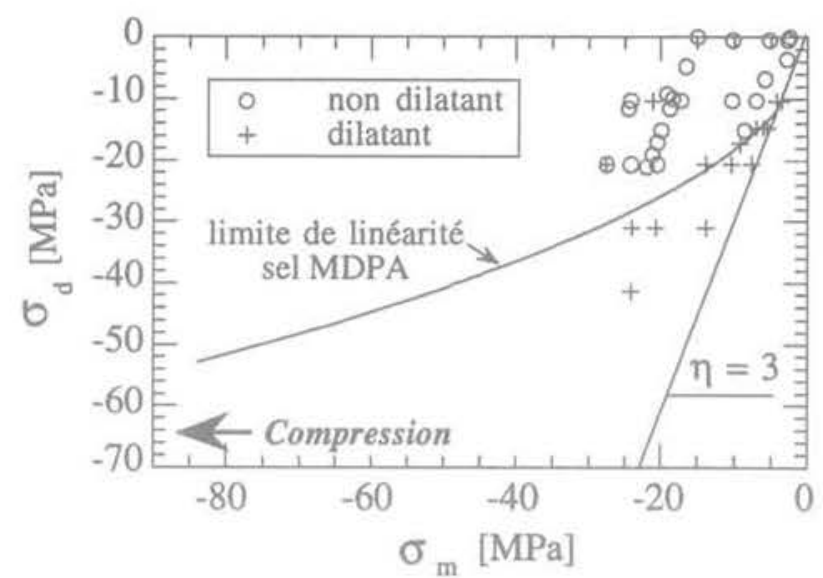

FG. 8 États de contraintes générant ou non de la dilatance au cours d'essais de fluage en compression (d'après Van Sambeek et al., 1993).

Stress state corresponding to dilatant or nondilatant creep tests.

lité de ce matériau. Malgré l'effet Bauschinger constaté plus haut (Fig. 5), et compte tenu des applications envisagées (calcul de structures souterraines), l'écrouissage est choisi comme isotrope.

\section{1}

\section{Critères de rupture et d'initiation de l'endommagement}

L'initiation de l'endommagement est, nous l'avons vu, plus précoce en extension qu'en compression. Le critère d'endommagement est identifié pour deux trajets de chargement, tandis que le critère de rupture n'est déterminé qu'en compression, puisque la rupture au sens du pic de résistance n'est pas révélée en extension.

Leur expression est généralisée à l'espace des contraintes, en les exprimant en fonction de la contrainte équivalente de Von Mises $\sigma_{\text {eq }}$ de la contrainte moyenne $\sigma_{\mathrm{m}}$ et de $\mathrm{J}_{\mathrm{m}}$

Le critère d'initiation de l'endommagement, projeté dans le plan $\left|\sigma_{m} ; \sigma_{e q}\right|$ possède une forme parabolique, tandis que le critère de rupture est plutôt linéaire. La rupture en extension n'étant pas apparue, nous supposons, afin de fixer le critère de rupture dans le cas $\mathrm{J}_{\mathrm{m}}=$ +1 , que pour rompre l'éprouvette en suivant ce type de trajet de chargement, il est nécessaire de pénétrer dans le domaine des tractions jusqu'à ce qu'une contrainte principale atteigne la valeur de la résistance en traction (voisine de $2 \mathrm{MPa}$ pour le sel gemme). Nous avons

Critère d'endommagement $\sigma_{e q}=\left(\frac{-\sigma_{m}}{Z\left(J_{m}\right)}\right)^{1 / 2}$

$\operatorname{avec} \mathrm{Z}\left(\mathrm{J}_{\mathrm{m}}\right)=\mathrm{h}\left(\mathrm{Z}_{\mathrm{e}}, \mathrm{Z}_{\mathrm{c}}, \mathrm{J}_{\mathrm{m}}\right) \mathrm{Z}_{e}=0,05 \mathrm{MPa} \mathrm{Z}_{\mathrm{c}}=0,03 \mathrm{MPa}$

Critère de rupture $\quad \mathrm{R}=\sigma_{\mathrm{eq}}+\mathrm{A}\left(\mathrm{J}_{\mathrm{m}}\right) \sigma_{\mathrm{m}}-\mathrm{B}\left(\mathrm{J}_{\mathrm{m}}\right)$ $\operatorname{avec} A\left(J_{m}\right)=h\left(A_{e^{\prime}} A_{c^{\prime}} J_{m}\right) \quad B\left(J_{m}\right)=h\left(B_{e^{\prime}} B_{e^{\prime}} J_{m}\right)$

$$
\begin{array}{ll}
A_{e}=3 / 2 & R_{e}=2 \mathrm{MPa} \\
A C=1,35 & B_{c}=17,7 \mathrm{MPa} \\
& B_{e}=\frac{3 R_{t}}{2} \mathrm{MPa}
\end{array}
$$



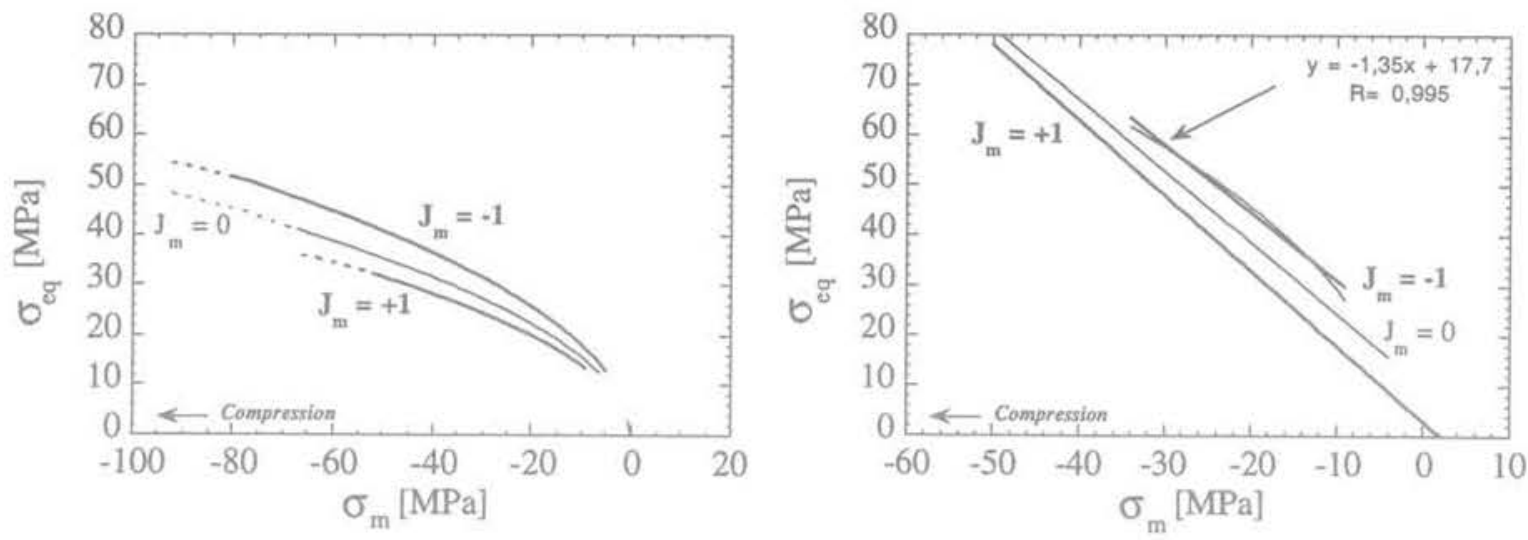

FIG. 9 Critères d'initiation de l'endommagement (à gauche) et de rupture (à droite) utilisés dans le modèle. Damage (left) and failure (right) criteria used in the model.

Les trajets de chargement suivis correspondent à $J_{m}$ $= \pm 1$. Afin de généraliser à toute valeur de $\mathrm{J}_{\mathrm{m}}$, comprise entre +1 et -1 , on utilise une fonction d'interpolation $h\left(x, y, J_{m}\right)$. Cette fonction est continue et sa dérivée par rapport a $\mathrm{J}_{\mathrm{m}} \mathrm{s}^{\prime}$ annule quand $\mathrm{J}_{\mathrm{m}}= \pm 1$ :

$$
h\left(x, y, J_{m}\right)=\frac{x-y}{2} \sin \left(\frac{\pi}{2} J_{m}\right)+\frac{x+y}{2}
$$

\section{2}

\section{Partition des déformations}

On suppose que les déformations linéarisées totales se décomposent en déformations élastiques $\varepsilon^{t}$ et en déformations plastiques $\underline{\underline{\varepsilon}}^{p}$ :

$$
\text { Déformations } \quad \underline{\underline{\varepsilon}}=\underline{\underline{\varepsilon}}^{\varepsilon}+\underline{\underline{\varepsilon}}^{p}
$$
avec :

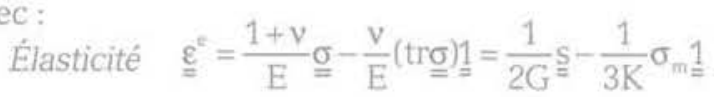

\section{3}

\section{Paramètre d'écrouissage}

Dans un premier temps, nous avons étudié le choix d'une variable d'écrouissage liée à la déformation volumique (directement liée à la perméabilité du sel). Les faibles variations de celle-ci sous fort confinement, ainsi qu'au début du chargement déviatorique (Fig. 6), ne permettent pas de reproduire correctement tous les phénomènes observés. Cela conduit à choisir un paramètre d'écrouissage $\xi$, unique, dépendant de la distorsion plastique $\gamma^{p}$, et traduisant un seul mécanisme d'écrouissage.

$$
\begin{aligned}
& \text { Écrouissage }
\end{aligned}
$$

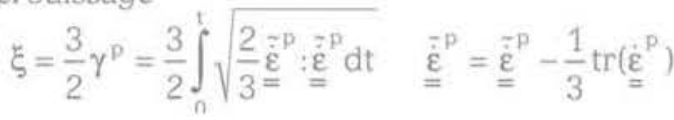

avec $\underline{\tilde{\varepsilon}}^{-p}=\begin{aligned} & \text { teneur déviateur des vitesses des déforma- } \\ & \text { tions linéarisées }\end{aligned}$

La surface de charge, le potentiel plastique et leurs évolutions en fonction de la distorsion plastique sont déterminés à partir des données expérimentales.

\section{4}

\section{Surface de charge}

Afin de déterminer la surface de charge, on reporte dans le plan $\left\{\sigma_{m} ; \sigma_{\mathrm{o}}\right\}$, pour différents essais, l'état de contrainte correspondant à plusieurs valeurs du paramètre d'écrouissage $\xi$ (Fig. 10).

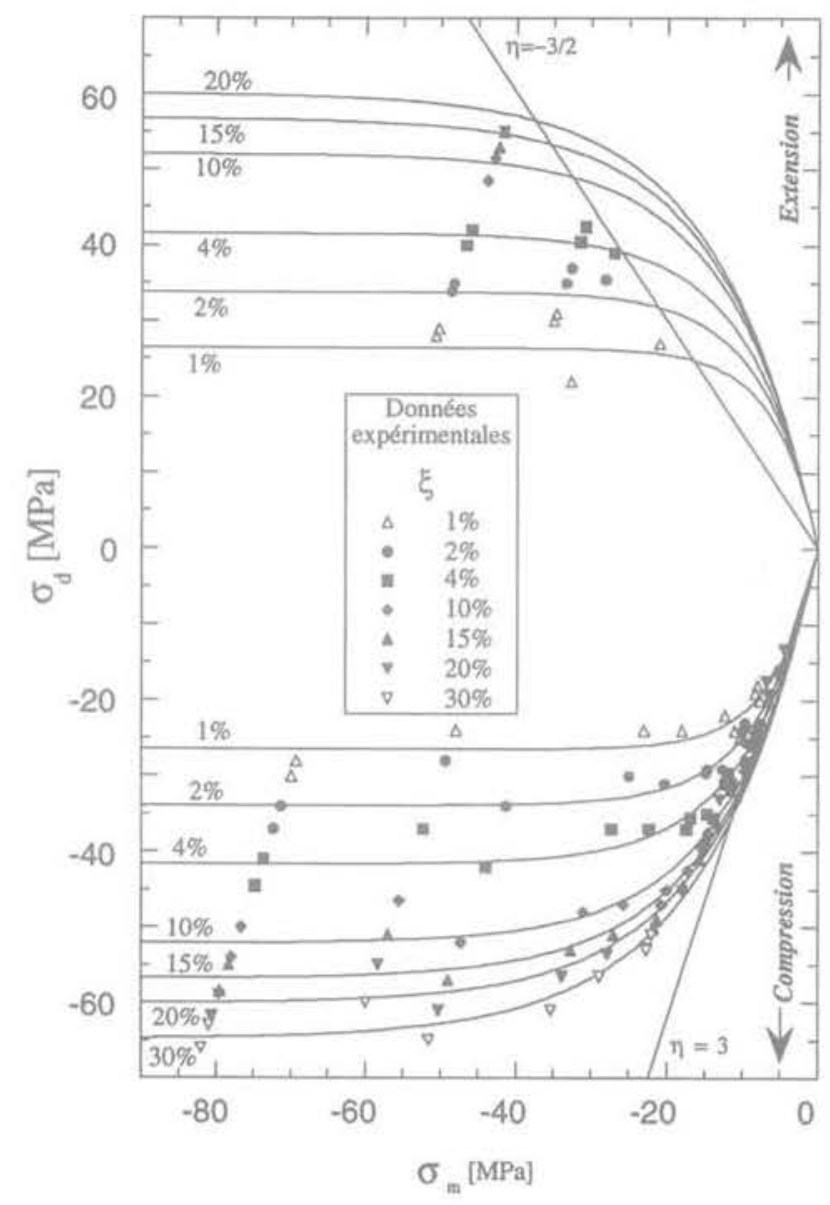

FiG. 10 États de contrainte et surface de charge pour différentes valeurs du paramètre d'écrouissage.

Stress and yield surface as a function of strain hardening parameter. 
On constate tant en compression qu'en extension, qu'une courbe de même expression peut convenir (6).

Surface de charge $\mathrm{F}(\underline{\underline{\sigma}}, \sigma)=\sigma_{\mathrm{eq}}-\sigma_{\mathrm{d}-}\left[1-\exp \left(\beta \frac{\sigma_{\mathrm{m}}}{\sigma_{\mathrm{d} m}}\right)\right]$

$$
\beta=4,5 \quad k_{1}=26,8 \mathrm{MPa}
$$

$\sigma_{\mathrm{d} m}=\mathrm{k}_{1} \log \left(\mathrm{k}_{2} \xi+1\right)+\mathrm{k}_{3}$

$\mathrm{k}_{\mathrm{a}}=$ seuil de plasticité initial pour $\sigma_{\mathrm{m}} \rightarrow-\infty$

$$
k_{2}=870 \quad 0<k_{3} \ll \ll 1
$$

Ce critère ne dépend pas de $J_{m}$. L'évolution du paramètre $\sigma_{\text {des }}$ correspond au résultat d'un essai réalisé dans le régime ductile, c'est-à-dire sous une pression de confinement très élevée.

\section{5}

\section{Potentiel plastique : analyse de l'angle de dilatance}

Les déformations volumiques irréversibles en cours d'essai, sont analysées par le biais de l'angle de dilatance.

Ce choix repose sur le fait que la direction d'écoulement plastique dépend de la nature des déformations plastiques à reproduire, en particulier des déformations volumiques irréversibles. La direction de l'écoulement plastique est donnée par la normale au potentiel plastique $G$ (si celui-ci existe), défini dans l'espace des contraintes. Pour de petites déformations, on écrit :

$$
\mathrm{d} \underline{\underline{\varepsilon}}^{\mathrm{p}}=\mathrm{d} \lambda \frac{\partial \mathrm{G}}{\partial \underline{\underline{\varepsilon}}} \quad \text { ou } \quad \underline{\underline{\varepsilon}}^{\mathrm{p}}=\dot{\lambda} \frac{\partial \mathrm{G}}{\partial \underline{\underline{\sigma}}}
$$

$$
\text { avec } \quad \mathrm{d} \lambda \geq 0 \text { si } \mathrm{F}=0 \text { et } \dot{\mathrm{F}}=0
$$

$$
\text { et } \quad \mathrm{d} \lambda=0 \text { si } \mathrm{F}<0
$$

Pour une loi associée, l'écoulement est normal et $\mathrm{F}=$ G. Cette situation n'est pas en général le cas des géomatériaux, pour lesquels se développent souvent des déformations volumiques irréversibles qui se tradujsent par des lois non associées $(F \neq G)$.

Si le matériau est isotrope dans son état initial (hypothèse plausible pour le sel gemme) et le demeure tant pendant la déformation élastique que pendant la déformation plastique, le potentiel plastique peut être exprimé en fonction de trois invariants des contraintes, par exemple de la contrainte moyenne $\sigma_{\text {, }}$, de la contrainte équivalente de Von Mises $\sigma_{\text {ca }}$ et du paramètre adimensionnel décrivant la géométrie des contraintes $\mathrm{J}_{\mathrm{m}}$ :

$$
\mathrm{G}=\mathrm{G}\left(\sigma_{\mathrm{m}}, \sigma_{\mathrm{eq}}, \mathrm{J}_{\mathrm{m}}\right)
$$

Rappelons que le paramètre de géométrie des contraintes $J_{m}$ est nul dans le cas d'un état de contrainte isotrope, mais aussi lorsque $\mathrm{J}_{3}$ (le troisième invariant du tenseur déviateur des contraintes) est nul. $\mathrm{J}_{\mathrm{m}}$ ne peut être calculé si $\sigma_{\text {eff }}\left(\right.$ ou $J_{2}$ ) est nul. Cette situation revient au cas de la contrainte isotrope, et donc $\mathrm{J}_{\mathrm{m}}=0$.

La dérivée du potentiel par rapport au tenseur des contraintes s'exprime alors:

$$
\frac{\partial G}{\partial \underline{\underline{\sigma}}}=\frac{\partial G}{\partial \sigma_{m}} \frac{\partial \sigma_{m}}{\partial \underline{\underline{\sigma}}}+\frac{\partial G}{\partial \sigma_{c o}} \frac{\partial \sigma_{i q}}{\partial \underline{\underline{\sigma}}}+\frac{\partial G}{\partial J_{m}} \frac{\partial J_{m}}{\partial \underline{\underline{\sigma}}}
$$

avec:

$$
\frac{\partial \sigma_{\mathrm{m}}}{\partial \underline{\underline{\sigma}}}=\frac{1}{3} \underline{\underline{1}} ; \frac{\partial \sigma_{\mathrm{eq}}}{\partial \underline{\underline{\sigma}}}=\frac{3}{2 \sigma_{\mathrm{eq}}} \underline{\underline{\mathrm{S}}} ; \frac{\partial \mathrm{J}_{\mathrm{m}}}{\partial \underline{\underline{\sigma}}}=\frac{9}{2 \sigma_{\mathrm{eq}}^{2}}\left[\frac{3}{\sigma_{\mathrm{eq}}} \mathrm{t}-\mathrm{J}_{\mathrm{m}} \mathrm{s}\right]
$$

où ț est le déviateur du carré du tenseur $\underline{\underline{\underline{s}}}$ déviateur des contraintes:

$$
\underline{t}=\frac{\partial J_{3}}{\partial \underline{\sigma}}=\underline{s}^{2}-\frac{2 J_{2}}{3} \underline{1}=\underline{\underline{s}} \underline{s}^{2}-\frac{2}{9} \sigma_{\text {e् }}^{2} \underline{1}
$$

On remarque que $t$ et s ont mêmes directions principales.

\section{4581}

\section{Angle de dilatance: cas général}

La normale non unitaire à la surface $G$ s'écrit dans les trois directions de l'espace $\left\{\sigma_{\mathrm{m},}, \sigma_{\mathrm{eq},}, \mathrm{J}_{\mathrm{m}} \mid\right.$ :

$$
\underline{n}=\left(\begin{array}{lll}
\frac{\partial G}{\partial \sigma_{m}} & \frac{\partial G}{\partial \sigma_{c q}} & \frac{\partial G}{\partial J_{m}}
\end{array}\right)
$$

La formule (8) permet d'obtenir :

$$
\begin{aligned}
& \dot{\varepsilon}_{\mathrm{v}}^{p}=\operatorname{tr} \underline{\underline{\varepsilon}}^{\dot{p}}=\underline{\underline{\varepsilon^{p}}}: 1=\lambda \frac{\partial \mathrm{G}}{\partial \sigma_{m}} \\
& \stackrel{\vec{\varepsilon}}{=}=\dot{\varepsilon}^{p}-\frac{1}{3} \dot{\varepsilon}_{v}^{p} 1=\dot{\lambda}\left[\frac{27}{2 \sigma_{\text {eq }}^{3}} \frac{\partial \mathrm{G}}{\partial J_{m}} t+\frac{3}{2 \sigma_{\text {eq }}}\left(\frac{\partial \mathrm{G}}{\partial \sigma_{\text {eq }}}-\frac{3 J_{m}}{\sigma_{\text {eq }}} \frac{\partial \mathrm{G}}{\partial J_{m}}\right) \leqq\right.
\end{aligned}
$$

avec $\dot{\varepsilon}_{v}^{n}=$ vitesse de déformation volumique irréversible.

On remarque que la vitesse d'évolution de la déformation volumique est proportionnelle à la composante de la normale dans la direction de l'axe $\sigma_{m}$. Il y aura dilatance ou contractance si cette composante est non nulle. On définit alors l'angle de dilatance compris entre la direction de la normale et la projection de la normale dans le plan $\sigma_{m}=0$ (Fig. 11). Cet angle est positif si les déformations volumiques irréversibles sont dilatantes.

La normale $\underline{n}$ au potentiel plastique projetée dans le plan $\sigma_{m}=0$ donne le vecteur $\underline{m}$. L'angle s'ouvrant de $\underline{m}$ vers n est l'angle de dilatance $\delta$. Il est positif s'il y a dilatance et négatif en cas de contractance.

L'angle de dilatance $\delta$ s'exprime en fonction des composantes de la normale $\mathrm{n}$ au potentiel plastique :

$$
\operatorname{tg} \delta=\frac{\frac{\partial G}{\partial \sigma_{m}}}{\sqrt{\left(\frac{\partial G}{\partial \sigma_{e q}}\right)^{2}+\left(\frac{\partial G}{\partial J_{m}}\right)^{2}}}
$$

\section{6?}

\section{Puissance volumique de dissipation plastique}

Écrivons la puissance volumique de dissipation plastique en petites déformations linéarisées :

$$
\begin{aligned}
& \underline{\sigma}: \dot{\varepsilon}^{p}=\dot{\lambda} \underline{\underline{\sigma}}: \frac{\partial G}{\partial \underline{\underline{\sigma}}}=\dot{\lambda} \underline{\underline{\sigma}}:\left[\frac{\partial G}{\partial \sigma_{m}} \frac{\partial \sigma_{m}}{\partial \underline{\underline{\sigma}}}+\frac{\partial G}{\partial \sigma_{c q}} \frac{\partial \sigma_{\mathrm{eq}}}{\partial \underline{\underline{\sigma}}}+\frac{\partial G}{\partial J_{m}} \frac{\partial J_{m}}{\partial \underline{\underline{\sigma}}}\right] \\
& =\lambda \underline{\underline{\sigma}}:\left[\frac{\partial \mathrm{G}}{\partial \sigma_{\mathrm{m}}} \frac{1}{3} 1+\frac{\partial \mathrm{G}}{\partial \sigma_{\mathrm{eq}}} \frac{3}{2 \sigma_{\mathrm{eq}}} \mathrm{s}+\frac{\partial \mathrm{G}}{\partial J_{m}} \frac{9}{2 \sigma_{\mathrm{eq}}^{2}}\left(\frac{3}{\sigma_{\mathrm{eq}}} \mathrm{t}-\mathrm{J}_{\mathrm{m}} \mathrm{s}\right)\right]
\end{aligned}
$$



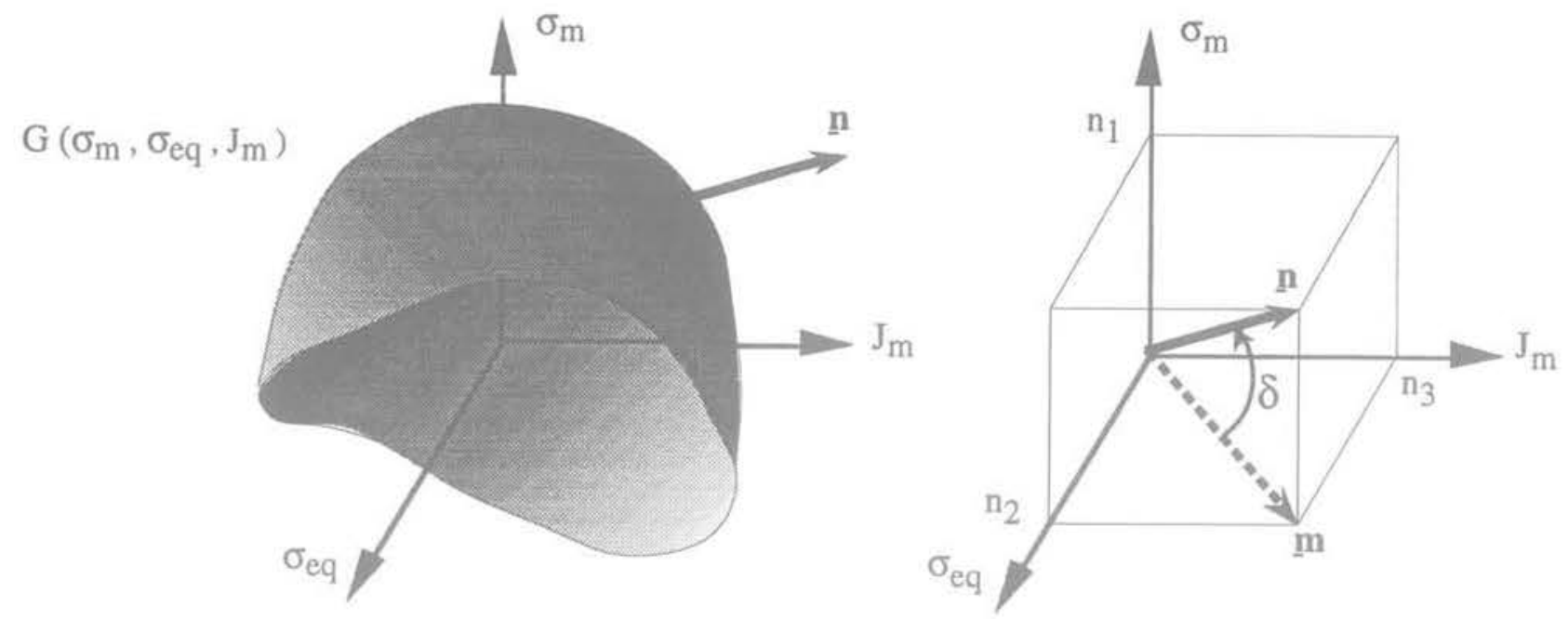

FIG.11 Potentiel plastique et angle de dilatance. Plastic potential and dilatancy angle.

Rappelons quelques calculs de produits contractés:

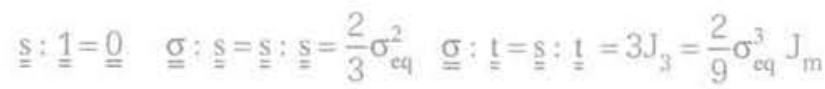

Il en résulte que le dernier terme de (16) s'annule, ce qui conduit à l'expression de la puissance volumique dissipée, pour un milieu isotrope, quel que soit le potentiel d'écoulement G :

$$
\underline{\underline{\sigma}}: \stackrel{\dot{\varepsilon}^{p}}{=}=\lambda\left[\sigma_{m} \frac{\partial G}{\partial \sigma_{m}}+\sigma_{e q} \frac{\partial G}{\partial \sigma_{m}}\right]
$$

On remarque que le troisième invariant du tenseur des contraintes n'intervient pas sur la dissimulation d'énergie mécanique lors de déformations plastiques dans un milieu isotrope.

En utilisant la formule (19) et le fait que $\mathrm{s}$ et $\mathrm{t}$ ont mêmes directions principales, on constate que le tenseur déviateur des déformations et le tenseur déviateur des contraintes ont eux aussi mêmes directions principales, ce qui est vrai en raison de l'isotropie supposée permanente du matériau. On peut ainsi définir l'orientation des tenseurs déviatoriques:

$$
\underline{\underline{\mathrm{n}}}=\frac{\underline{\underline{\underline{\mathrm{S}}}}}{\sqrt{\underline{\underline{\underline{S}}}: \underline{\underline{\underline{S}}}}}=\frac{\frac{\overrightarrow{\tilde{\varepsilon}}}{\underline{\underline{\varepsilon}}}}{\sqrt{\underline{\underline{\underline{\varepsilon}}}: \frac{\vec{\varepsilon}}{\underline{\varepsilon}}}}
$$

Le produit contracté du tenseur par lui-même donne $\underline{\underline{t}}: \underline{\mathrm{t}}=\operatorname{tr}\left(\underline{\underline{s}}^{4}\right)-\frac{4}{27} \sigma_{\text {en }}^{4}$

En constatant que la puissance volumique de dissipation plastique s'écrit aussi :

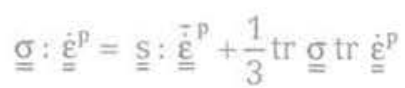

et en introduisant le taux de distorsion plastique :

$\dot{\gamma}^{p}=\sqrt{\frac{2}{3} \underline{\underline{\varepsilon^{p}}}: \underline{\underline{\varepsilon}}^{p}}=\dot{\lambda}\left[\frac{g}{\sigma_{\mathrm{eq}}^{2}}\left(\frac{81}{2 s_{\mathrm{eq}}^{4}} \operatorname{tr}\left(\underline{\underline{s}}^{4}\right)-2-J_{\mathrm{m}}^{2}\right)\left(\frac{\partial \mathrm{G}}{\partial J_{\mathrm{m}}}\right)^{2}+\left(\frac{\partial \mathrm{G}}{\partial \sigma_{\mathrm{eq}}}\right)^{2}\right]^{1 / 2}$ il vient :

$$
\begin{aligned}
& \underline{\underline{\sigma}}: \dot{\underline{\varepsilon}}^{\mathrm{p}}=\sqrt{\frac{3}{2} \underline{\underline{S}} ; \underline{\underline{S}}} \dot{\gamma}^{p}+\frac{1}{3} \operatorname{tr} \underline{\underline{g}} \dot{\varepsilon}^{p} \\
& \underline{\underline{\underline{\sigma}}}: \dot{\underline{\varepsilon}}^{p}=\sigma_{\mathrm{eq}} \dot{\gamma}^{p}+\sigma_{\mathrm{m}} \dot{\varepsilon}_{v}^{p}
\end{aligned}
$$

On veillera évidemment au respect de la positivité de cette dissipation volumique.

\section{5 .3}

\section{Angle de dilatance : cas de l'essai triaxial axisymétrique}

Dans le cas de l'essai trixial axisymétrique, $\mathrm{J}_{\mathrm{m}}=+1$ en extension, et - 1 en compression ; le tenseur déviateur des contraintes s'écrit :

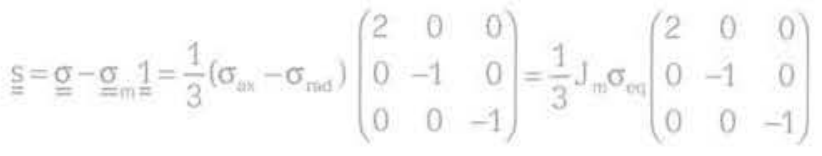

La contrainte équivalente de Von Mises se confond avec celle de Tresca :

$$
\sigma_{\mathrm{eq}}=\left|\sigma_{\mathrm{ax}}-\sigma_{\mathrm{rid}}\right|
$$

le tenseur déviateur du carré du déviateur des contraintes se simplifie :

$$
\underline{\underline{t}}=\frac{1}{3} J_{\mathrm{m}} \sigma_{\mathrm{rq}} \underline{\mathrm{S}}
$$

ce qui entraîne l'indépendance de la géométrie de contrainte $\mathrm{J}_{\mathrm{m}}$ avec le tenseur $\underline{\underline{\sigma}}$ :

$$
\frac{\partial J_{m}}{\partial \sigma}=\underline{\underline{0}}
$$

le tenseur déviateur du taux de déformation plastique se simplifie :

$$
\underline{\underline{\varepsilon}}=\lambda \frac{\partial \mathrm{G}}{\partial \sigma_{\mathrm{eq}}} \frac{3}{2 \sigma_{\mathrm{eq}}} \stackrel{\underline{S}}{\mathrm{~s}}
$$


sa première composante s'écrit :

$$
\tilde{\varepsilon}_{11}^{p}=\frac{2}{3}\left(\dot{\varepsilon}_{\mathrm{ax}}^{\mathrm{p}}-\dot{\varepsilon}_{\mathrm{rad}}^{\mathrm{p}}\right)
$$

le taux de distorsion plastique devient:

$$
\dot{\gamma}^{p}=\lambda\left|\frac{\partial \mathrm{G}}{\partial \sigma_{\text {eq }}}\right|=\frac{2}{3}\left|\dot{\varepsilon}_{\text {ax }}^{p}-\dot{\varepsilon}_{\text {rad }}^{p}\right|=\left|\tilde{\varepsilon}_{11}^{p}\right|
$$

On trouve finalement, en éliminant le multiplicateur plastique $\dot{\lambda}$ entre (16) et (33), que le rapport entre les deux premières composantes de la normale au potentiel plastique est proportionnel à la déformation volumique rapportée au taux de distorsion plastique :

$$
\frac{\frac{\partial G}{\partial \sigma_{m}}}{\left|\frac{\partial G}{\partial \sigma_{e q}}\right|}=\frac{\dot{\varepsilon}_{v}^{p}}{\dot{\gamma}^{p}}
$$

Pour les trajets de chargement accessibles à l'essai triaxial axisymétrique, la valeur de $J_{m}$ est +1 en extension et - 1 en compression. Il est alors difficile de calculer le gradient du potentiel plastique G par rapport à $\mathrm{J}_{\mathrm{m}}$ sur la base de ces seuls types d'expériences. D'autres trajets de chargement conviendraient d'être utilisés, par exemple avec un appareil triaxial vrai. Nous imposerons arbitrairement nul le gradient de $\mathrm{G}$ par rapport à $\mathrm{J}_{\mathrm{m}}$ :

$$
\frac{\partial G}{\partial J_{m}}=0 \text { si } J_{m}^{2}=1
$$

Cette hypothèse permet de simplifier la définition (18) de l'angle de dilatance, qui devient:

$$
\operatorname{tg} \delta=\frac{\frac{\partial G}{\partial \sigma_{m}}}{\left|\frac{\partial G}{\partial \sigma_{e q}}\right|}
$$

ou encore, d'après (31) :

$$
\delta=\operatorname{arctg}\left(\frac{\partial \varepsilon_{v}^{p}}{\partial \gamma^{p}}\right)
$$

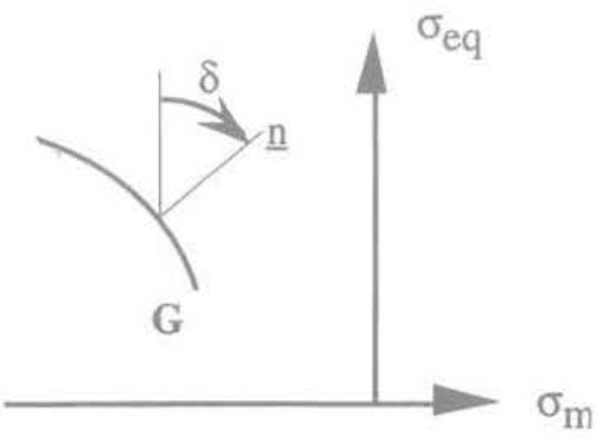

Définition simplifiée de l'angle de dilatance $\delta$ pour un essai triaxial axisymétrique.

Simplified definition of the dilatancy angle for a triaxial axisymetric test.

Cet angle $\delta$ s'annule pour une déformation volumique isochore, est positif en cas de dilatance plastique et négatif pour une contractante plastique. Il donne, dans le plan (contrainte moyenne ; contrainte équivalente de Von Mises) l'orientation de la normale au potentiel plastique. Il est positif dans le sens rétrograde avec la convention des compressions négatives.

Si nous supposons de plus que : $\frac{\partial G}{\partial \sigma_{e q}} \geq 0$

cela entraine : $\frac{\partial G}{\partial \underline{\underline{\sigma}}}=\frac{\partial G}{\partial \sigma_{\mathrm{eq}}}\left(\operatorname{tg} \delta \frac{\partial \sigma_{\mathrm{m}}}{\partial \underline{\underline{\sigma}}}+\frac{\partial \sigma_{\mathrm{eq}}}{\partial \underline{\underline{\sigma}}}\right)$ et finale-
ment:

$$
\frac{\partial \mathrm{G}}{\partial \underline{\underline{\sigma}}}=\frac{\partial \mathrm{G}}{\partial \sigma_{\mathrm{eq}}}\left[\frac{\operatorname{tg} \delta}{3} \underline{\underline{1}}+\frac{3}{2 \sigma_{\mathrm{eq}}} \stackrel{\underline{\mathrm{s}}}{\mathrm{g}}\right]
$$

Si le potentiel plastique s'exprime sous la forme $G$ $=\sigma_{\mathrm{eq}}-\mathrm{f}\left(\sigma_{\mathrm{m}}, \xi\right)$ ) alors $\frac{\partial \mathrm{G}}{\partial \sigma_{\mathrm{eq}}}=1$ (car $\sigma_{e q}$ et $\sigma_{m}$ sont deux variables indépendantes) et il reste :

$$
\frac{\partial \mathrm{G}}{\partial \underline{\underline{\sigma}}}=\frac{\operatorname{tg} \delta}{3} \stackrel{\underline{1}}{=}+\frac{3}{2 \sigma_{\mathrm{mq}}} \stackrel{\mathrm{s}}{=}
$$

Par cette dernière relation, on voit que la direction d'écoulement ne dépend que de l'état de contrainte et de l'angle de dilatance pour les chargements en conditions triaxiales axisymétriques. C'est donc au travers de l'angle de dilatance que la direction d'écoulement dépend éventuellement de la variable d'écrouissage. Il faut alors étudier l'évolution de l'angle de dilatance en fonction de l'état de contrainte, mais aussi en fonction du paramètre d'écrouissage.

Pour l'analyse d'essais triaxiaux axisymétriques, l'angle de dilatance est calculé par:

$$
\delta=\operatorname{arctg}\left(\frac{3}{2} \frac{\sigma_{\mathrm{d}}}{\sigma_{\mathrm{a}}} \mid \frac{\partial \varepsilon_{\mathrm{v}}^{\mathrm{p}}}{\partial \varepsilon_{\mathrm{d}}^{\mathrm{p}}}\right)
$$

La contrainte déviatorique $\sigma_{\mathrm{d}}=\sigma_{\mathrm{ax}}-\sigma_{\mathrm{rad}}$ est de même signe que la vitesse de déformation plastique déviatorique $\dot{\varepsilon}_{\mathrm{p}}^{p}=\dot{\varepsilon}_{\mathrm{ax}}^{p}-\dot{\varepsilon}_{\mathrm{rud}}^{p}$. On peut ainsi dissocier les résultats d'essais triaxiaux axisymétriques de compression et d'extension, en représentant les surfaces de charge dans le plan icontrainte moyenne; contrainte

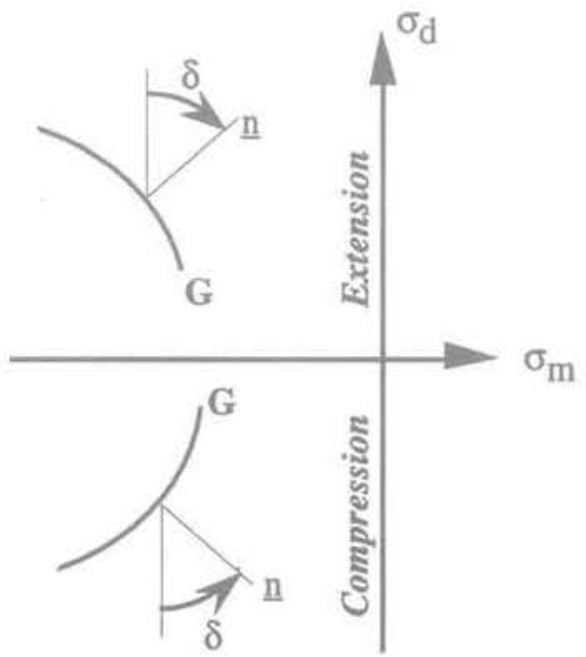

FG. 13 Angle de dilatance en compression et en extension dans le plan (contrainte moyenne; contrainte déviatorique]. Dilatancy angle for compression or extension loading, in (mean stress ; deviatoric stress) plane. 
déviatorique). Dans le cas de la compression, $\sigma_{\mathrm{d}} \leq 0$ et l'angle de dilatance est alors défini positif dans le sens trigonométrique (Fig. 13), c'est-à-dire en cas de dilatance.

Au cours d'un essai triaxial axisymétrique, il est ainsi possible de connaître la direction d'écoulement à tout instant, et en particulier pour des valeurs déterminées du paramètre d'écrouissage. On peut alors déterminer la forme du potentiel plastique et son évolution. tout en gardant en mémoire les deux conditions (35) et (38) imposées sur les composantes du gradient du potentiel plastique $\mathrm{G}$ en fonction de la contrainte équivalente $\sigma_{\text {eq }}$ et de la géométrie des contraintes $J_{m}$.

Cela permet de tracer les directions d'écoulement et de constater que celui-ci ne peut être associé pour toutes les valeurs de la pression de confinement. La figure 14 fait apparaitre en particulier que pour des contraintes moyennes de l'ordre de $15 \mathrm{MPa}$, la normale à la surface de charge et la direction d'écoulement ne sont pas colinéaires. Le modèle élastoplastique sera donc effectivement non standard.

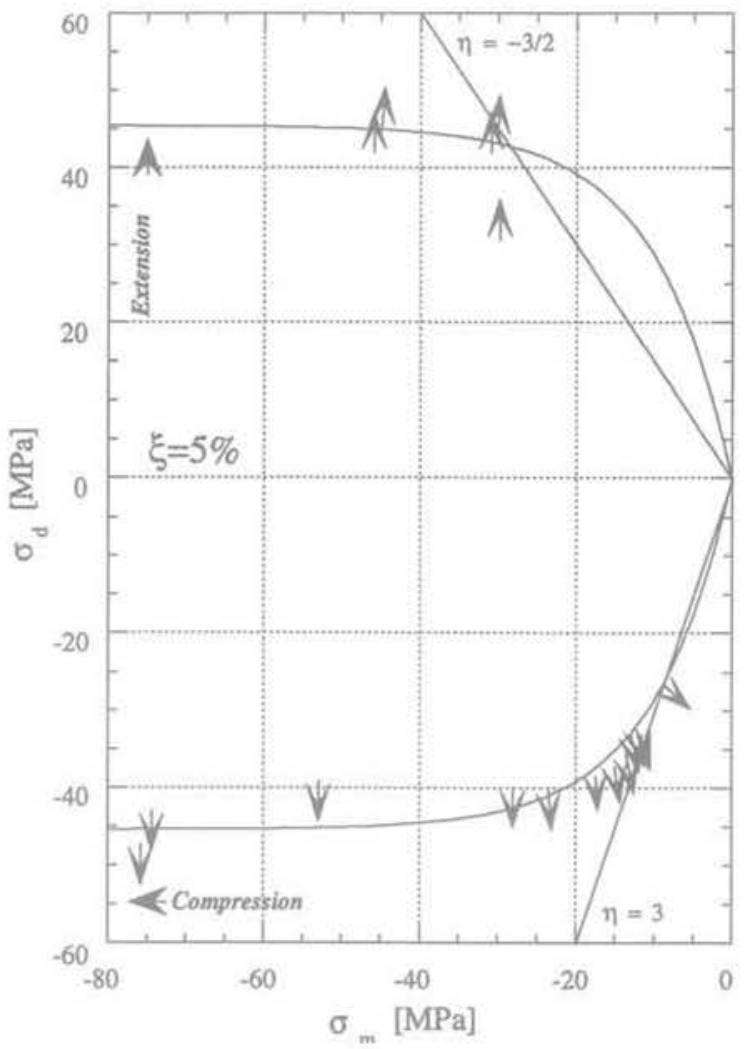

FIG.14 Directions d'écoulement pour une valeur donnée du paramètre d'écrouissage $\xi$. Vectors of plastic strain for a given value of the hardening parameter $\xi$.

\subsection{4}

\section{Évolution de l'angle de dilatance}

Au cours de la déformation plastique, l'angle de dilatance évolue. Le cas d'un angle de dilatance constant réduit le potentiel plastique à une droite dans le plan (contrainte moyenne ; contrainte déviatorique) (Senseny et al., 1983). Dans le cas étudié, nous constatons que l'angle de dilatance varie à la fois en fonction de la contrainte moyenne et de la distorsion plastique. Ceci est visible sur la figure 15 , où est présenté un exemple d'évolution de la contrainte déviatorique et de l'angle de dilatance en fonction du paramètre d'écrouissage.

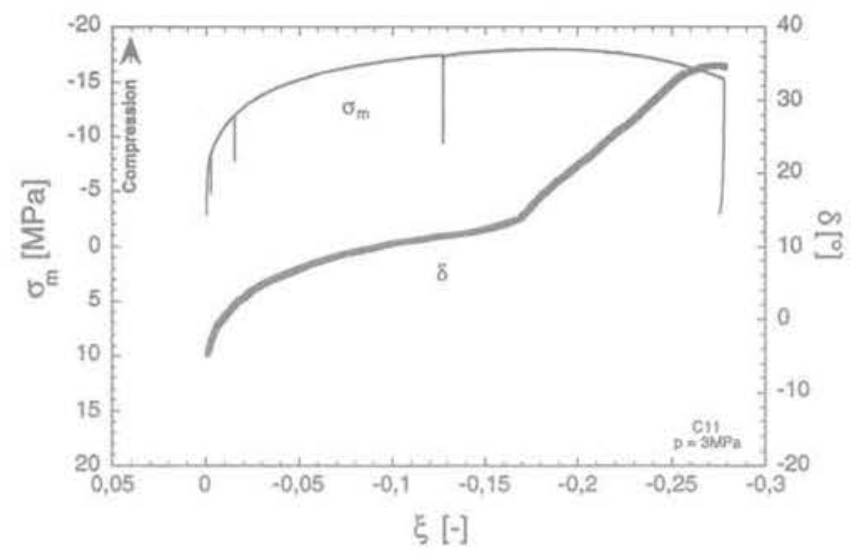

FG. 15 Évolution au cours d'un essai de compression triaxiale de la contrainte moyenne et de l'angle de dilatance en fonction du paramètre d'écrouissage $\xi$. Evolution of mean stress and dilatancy angle during a triaxial compressive test, as a function of the hardening parameter $\xi$.

Afin d'évaluer la dépendance de l'angle envers la contrainte moyenne et le paramètre d'écrouissage, on reporte, sur un graphique, la valeur de la tangente de l'angle de dilatance et la valeur de la contrainte moyenne (un exemple est donné sur la figure 16 pour une valeur du paramètre d'écrouissage de $10 \%$ ). Chaque point correspond à un essai différent.

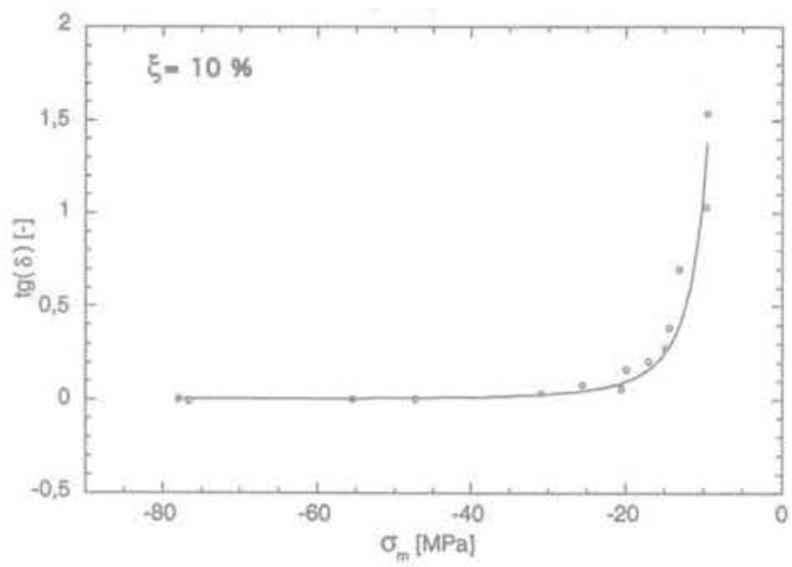

76. 16 Évolution de la tangente de I'angle de dilatance en fonction de la contrainte moyenne pour une valeur du paramètre d'écrouissage de $10 \%$.

Dilatancy angle as a function of mean stress for a given value of hardening parameter equal to $10 \%$.

Ce travail est répété tant en compression qu'en extension, pour différentes valeurs du paramètre d'écrouissage.

Nous proposons qu'une courbe identique à une translation près puisse décrire l'ensemble des cas étudiés en compression et en extension. Elle doit vérifier la présence d'une asymptote horizontale pour les contraintes moyennes infinies négatives, et une asymp- 
tote verticale pour une valeur de la contrainte moyenne à déterminer. Cette courbe est de la forme :

$$
\operatorname{tg}(\delta)=\left[\frac{b}{-\sigma_{m}+a}\right]^{s}
$$

Le paramètre « a $)$ est la valeur asymptotique de la contrainte moyenne pour laquelle la tangente de l'angle de dilatance tend vers l'infini. Dans ce cas (imaginaire) $\delta=\pi / 2$, il n'y a plus que des déformations volumiques irréversibles, les déformations déviatoriques étant nulles. Le traitement des données expérimentales permet de fixer les paramètres à :

$$
\mathrm{n}=5 / 2 \quad \mathrm{~b}=6,31 \mathrm{MPa}\left(=100^{2 / 9}\right) \quad \mathrm{a}=\mathrm{a}(\xi)
$$

Déduite des courbes d'interpolation (39), l'évolution de « a » avec la déformation déviatorique irréversible (le paramètre d'écrouissage ફ̧) donne en compression (Fig. 17) :

$$
\mathrm{a}=\frac{1}{\left(\mathrm{H}_{c}+\xi\right)^{\mathrm{m}}}-\mathrm{C}_{\mathrm{c}} \xi-\mathrm{D}_{\mathrm{c}}
$$

avec: $m=100 \mathrm{Hc}=0,964 \mathrm{C}_{\mathrm{c}}=38,82 \mathrm{MPa} \mathrm{D}_{\mathrm{c}}=0 \mathrm{MPa}$

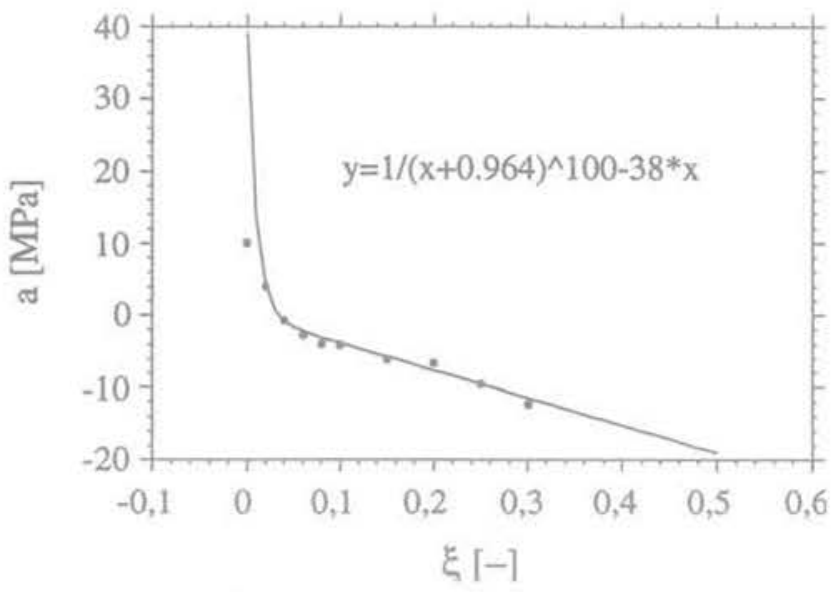

FG. 17 Evolution, en compression de l'abscisse de l'asymptote verticale de la courbe $\operatorname{tg}(\delta)=\mathrm{f}$ $\left(\sigma_{m}\right)$ en fonction de $\xi$.

" $a$ " as a function of $\xi$.

Bien que plus délicate à établir, l'évolution de " a » en extension revêt la même nature d'équation qu'en compression, à quelques nuances près reproduites sur les constantes:

$$
\mathrm{a}=\frac{1}{\left(\mathrm{H}_{\mathrm{e}}+\xi\right)^{\mathrm{m}}}-\mathrm{C}_{e} \xi-\mathrm{D}_{e}
$$

avec : $m=100 \mathrm{H}_{e}=0,964 \mathrm{C}_{\mathrm{e}}=60 \mathrm{MPa} \mathrm{D}_{\mathrm{e}}=10 \mathrm{MPa}$

Nous constatons que le paramètre " a » ne suit pas exactement la même évolution avec le paramètre d'écrouissage en compression et en extension. L'expression de « a » est généralisée par :

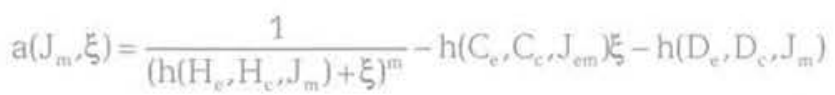

Le potentiel plastique s'exprime à partir de (36), (42), et (45), à une fonction près indépendante de la contrainte, par :

$$
\begin{array}{r}
\text { Potentiel plastique } \mathrm{G}=\sigma_{\mathrm{nq}}-\frac{\mathrm{b}^{\mathrm{n}}}{(1-\mathrm{n})\left[-\sigma_{\mathrm{m}}+\mathrm{a}\left(\mathrm{J}_{\mathrm{m}}, \xi\right)\right]^{\mathrm{n}-1}} \\
n=5 / 2 \quad b=6,31 \mathrm{MPa}
\end{array}
$$

La direction d'écoulement plastique est ainsi obtenue :

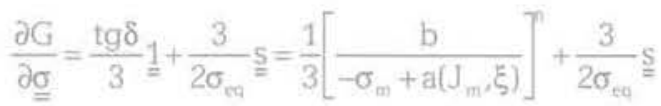

On vérifie bien que la dissipation volumique plastique est positive, en utilisant (27).

A titre d'illustration, sur la figure 18 est représentée Yallure de la surface de charge et du potentiel plastique pour un essai triaxial de compression, en indiquant, par un vecteur, la direction de l'écoulement pour deux valeurs du paramètre d'écrouissage $\xi$. Le trajet de chargement est celui d'un essai de compression sous $2 \mathrm{MPa}$ de pression de confinement. Il suit la droite de pente $\eta=-3$.

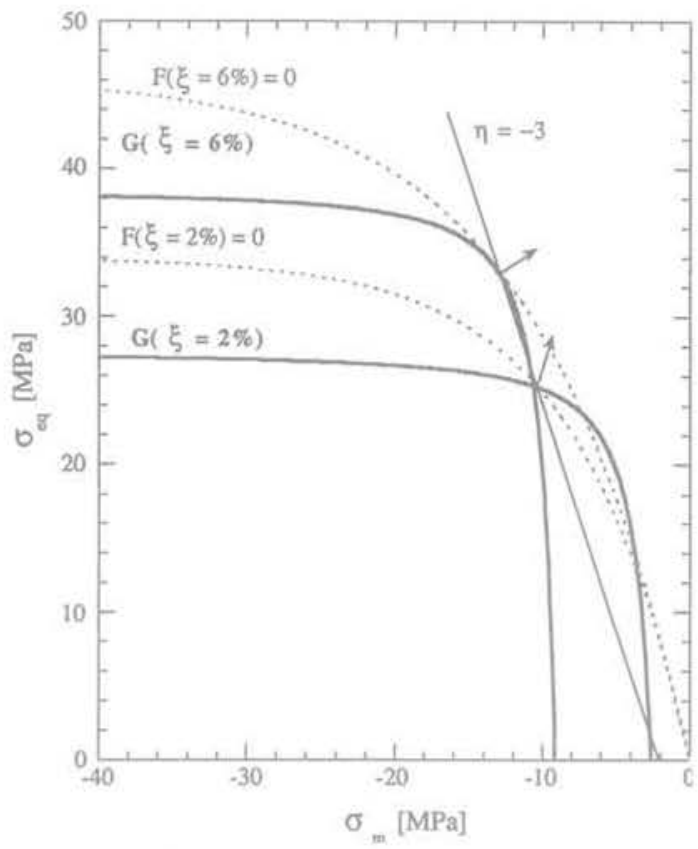

FIG. 18 Evolution de la surface de charge $F$ et du potentiel plastique $\mathrm{G}$ au cours d'un essa triaxial.

Evolution of yield surface and plastic potential during a triaxial test.

Pour implanter numériquement le modèle, il est nécessaire de connaître les dérivées suivantes:

Dérivées

$\frac{\partial G}{\partial \sigma_{e q}}=1 ; \frac{\partial G}{\partial \sigma_{m}}=\frac{b^{n}}{\left[-\sigma_{m}+a\left(J_{m}, \xi\right)\right]^{n}} ; \frac{\partial G}{\partial J_{m}}=-\frac{\partial G}{\partial \sigma_{m}} \frac{\partial a\left(J_{m} \cdot \xi\right)}{\partial J_{m}}$

$\frac{\partial \mathrm{a}\left(\mathrm{J}_{m}, \xi\right)}{\partial \mathrm{J}_{m}}=-\frac{\partial \mathrm{h}\left(\mathrm{H}_{\mathrm{c}}, \mathrm{H}_{c}, \mathrm{~J}_{\mathrm{m}}\right)}{\partial \mathrm{J}_{m}} \frac{\mathrm{m}}{\left(\mathrm{h}\left(\mathrm{H}_{x}, \mathrm{H}_{s}, \mathrm{~J}_{\mathrm{m}}\right)+\xi\right)^{m+1}}-\frac{\partial \mathrm{h}\left(\mathrm{D}_{v}, \mathrm{D}_{\mathrm{c}}, \mathrm{J}_{\mathrm{m}}\right)}{\partial \mathrm{J}_{\mathrm{m}}}$

$$
\frac{\partial h\left(x, y, J_{m}\right)}{\partial J_{m}}=\pi \frac{x-y}{4} \cos \left(\frac{\pi}{2} J_{n}\right)
$$




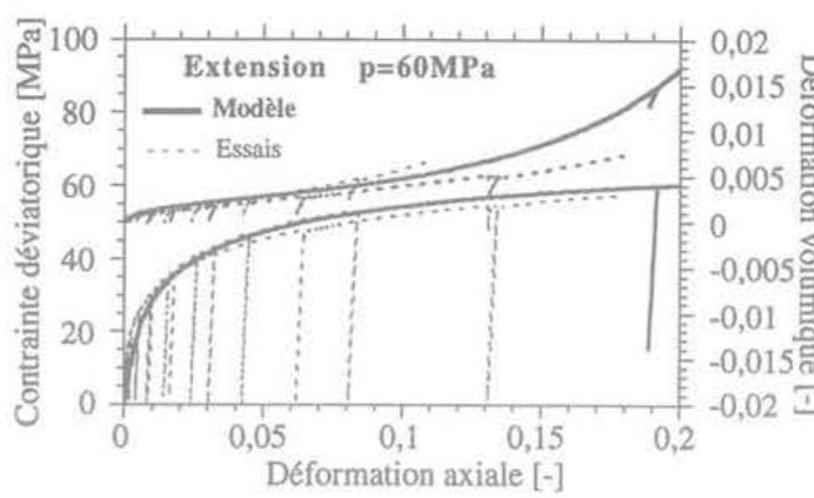

FiG. 19 Résultats expérimentaux et modélisation. Results of experiment and modelling.

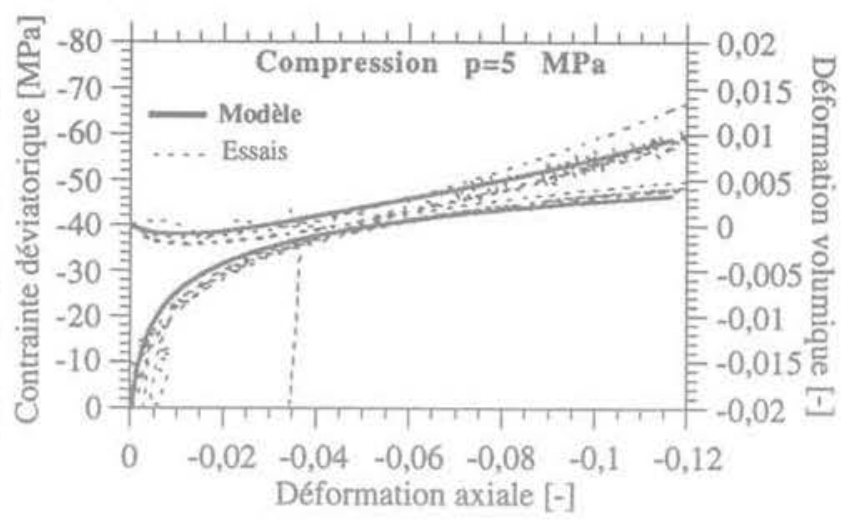

L'écriture tridimensionnelle complète du modèle, incluant les surfaces d'endommagement et de rupture, nécessite 15 jeux d'équations $(2,3,4,5,6,7,8,9,10,11$. $45,46,48,49,50$ ) et 22 paramètres dont 19 sont indépendants, tous étant déduits des expériences. Le nombre de paramètres peut être réduit, si cela s'avère justifié, en fonction du problème posé.

\section{6}

\section{Reproduction d'essais}

Programmé dans le code GEOMEC du laboratoire, ce modèle permet de retrouver d'une manière satisfaisante, l'évolution de la dilatance tant en compression qu'en extension, ainsi que les courbes d'écrouissage. Des exemples sont donnés sur la figure 19.

\section{7}

\section{Application à une structure souterraine}

Le sel gemme est utilisé dans de nombreux cas comme roche hôte de stockage souterrain, notamment d'hydrocarbures. L'apparition d'endommagement au voisinage d'une cavité de stockage peut nuire à la sûreté du stockage (Bêrest et Brouard, 1995). Une analyse élastique permet d'évaluer rapidement les conditions d'apparition de l'endommagement. Les résultats sur des cavités cylindrique et sphérique sont comparés, avant d'aborder le calcul complet d'une cavité cylindrique en déformations planes, en utilisant le modèle proposé tenant compte de l'endommagement.

TABLEAU1 Analyse élastique d'une cavité cylindrique et d'une cavité sphérique. Elastic analysis of cylindrical and spherical cavities.

\begin{tabular}{|c|c|c|}
\hline & Cavité cylindrique & Cavité sphérique \\
\hline$\sigma_{\mathrm{rr}}=$ & $-\mathrm{p}\left(1-\frac{1}{\mathrm{r}^{2}}\right)$ & $-\mathrm{p}\left(1-\frac{1}{\mathrm{r}^{3}}\right)$ \\
\hline$\sigma_{\theta \theta}=$ & $-\mathrm{p}\left(1+\frac{1}{\mathrm{r}^{2}}\right)$ & $-\mathrm{p}\left(1+\frac{1}{2 \mathrm{r}^{3}}\right)$ \\
\hline & $\sigma_{\mathrm{zz}}=-\mathrm{p}$ & $\sigma_{\phi \phi}=-\mathrm{p}\left(1+\frac{1}{2 \mathrm{r}^{3}}\right)$ \\
\hline$\sigma_{\mathrm{m}}=$ & $-\mathrm{p}$ & $-\mathrm{p}$ \\
\hline$\sigma_{\mathrm{eq}}=$ & $\sqrt{3} \frac{\mathrm{p}}{\mathrm{r}^{2}}$ & $\frac{3}{2} \mathrm{p}$ \\
\hline $\mathrm{J}_{\mathrm{m}}=$ & 0 & +1 \\
\hline $\begin{array}{c}\text { relation } \sigma_{\mathrm{eq}}=\mathrm{f}\left(\sigma_{\mathrm{m}}\right) \text { en paroi } \\
{[\mathrm{A}]}\end{array}$ & $\sigma_{\mathrm{eq}}=-\sqrt{3} \sigma_{\mathrm{m}}$ & $\sigma_{\mathrm{eq}}=\frac{-3}{2} \sigma_{\mathrm{m}}$ \\
\hline $\begin{array}{c}\text { Critère d'endommagement } \\
{[\mathrm{B}]}\end{array}$ & $\sigma_{\mathrm{eq}}=\left(\frac{-\sigma_{\mathrm{m}}}{0,04}\right)^{1 / 2}$ & $\sigma_{\mathrm{eq}}=\left(\frac{-\sigma_{\mathrm{m}}}{0,05}\right)^{1 / 2}$ \\
\hline $\begin{array}{c}\text { risque d'endommagement en paroi } \\
\text { (intersection de }[\mathrm{A}] \text { et }[\mathrm{B}])\end{array}$ & $8,3 \mathrm{MPa}(\approx 375 \mathrm{~m})$ & $8,9 \mathrm{MPa}(\approx 400 \mathrm{~m})$ \\
\hline $\begin{array}{c}\text { Critère de rupture } \\
{[\mathrm{C}]}\end{array}$ & $\sigma_{\mathrm{eq}}=-1,426 \mathrm{~m}+10,3$ & $\sigma_{\mathrm{eq}}=-1,5 \sigma_{\mathrm{m}}+3$ \\
\hline $\begin{array}{c}\text { risque de rupture en paroi } \\
\text { (intersection de }[\mathrm{A}] \text { et }[\mathrm{C}])\end{array}$ & $33,6 \mathrm{MPa}(\approx 1600 \mathrm{~m})$ & jamais \\
\hline
\end{tabular}




\section{Analyse élastique}

Avant le creusement, les cavités se trouvent dans un état de contrainte isotrope (hypothèse plausible pour le sel gemme, matériau dépourvu de tout seuil de viscoplasticité) caractérisé par la pression lithostatique $\mathrm{P}\left(\sigma^{0-}=-\mathrm{p} 1\right.$; . Le creusement de la cavité se traduit par une décharge brutale de la contrainte radiale en paroi de la structure $\sigma_{r T}^{0+}+(\mathrm{r}=1)=0$ (la cavité est de rayon unité).

Les conditions d'apparition de l'endommagement et de la rupture sont présentées sur le tableau I, Il faut cependant se garder de conclure à l'absence de tout risque de rupture pour les cavernes de la forme sphérique dans le sel. Cette situation tient essentiellement au critère de rupture employé. Celui-ci dépend d'une part de la contrainte moyenne (et non des contraintes principales extrêmes, comme c'est le cas du critère de Coulomb par exemple). D'autre part, le critère de rupture en extension a été défini en confondant la rupture en extension avec la rupture en traction (hypothèse à vérifier par des essais appropriés). II n'en reste pas moins que le risque de rupture pour une cavité sphérique est certainement plus faible que celui d'une cavité cylindrique. Le facteur de concentration de la contrainte orthoradiale étant plus élevé pour le cylindre que pour la sphère, la forme de cette dernière lui assure une meilleure stabilité mécanique.

\section{7 .2}

\section{Analyse élastoplastique}

L'analyse présentée ci-dessus en élasticité est complétée par un calcul en élastoplaticité. Celui-ci ne pouvant être mené analytiquement à partir du modèle proposé, le code de calcul aux éléments finis GEOMEC a été utilisé à cette fin. La loi de comportement et ses paramètres sont identiques à ceux déjà présentés.

Le calcul a été effectué dans le cas d'une cavité cylindrique de rayon unité soumise à l'infini (100 rayons) à une pression lithostatique de $25 \mathrm{MPa}$ (profondeur voisine de $1000 \mathrm{~m}$ ). Une tranche radiale du massif a été modélisée sous la double hypothèse de symétrie axiale et déformations planes. Elle est formée de 50 éléments isoparamétriques à 9 nœuds constituant au total 303 nœuds.

On retrouve naturellement l'allure typique de la répartition des contraintes autour d'une cavité circulaire (Fig. 20) : la contrainte orthoradiale passe par un minimum (en valeur absolue) à une certaine distance de la paroi tandis que la contrainte axiale et aussi la contrainte moyenne (Fig. 21) se déchargent en valeur absolue près de la paroi. Ce phénomène se traduit par une redistribution plastique de l'état des contraintes dans le massif de sorte que la proximité de l'ouvrage se trouve plutôt en extension (le paramètre $\mathrm{J}_{\mathrm{m}}$ repéré sur la figure 21 vaut 0,4 en élastoplasticité, il est nul en élasticité).

L'évolution spatiale des critères d'endommagement et de rupture présentée sur la figure 22 fait apparaître que la zone endommagée reste très localisée autour de l'ouvrage et s'arrête à environ deux rayons de la galerie. Cela est conforme aux résultats de Stormont et al. (1991) qui montrent par leurs mesures in situ une variation significative de la perméabilité entre la paroi et une

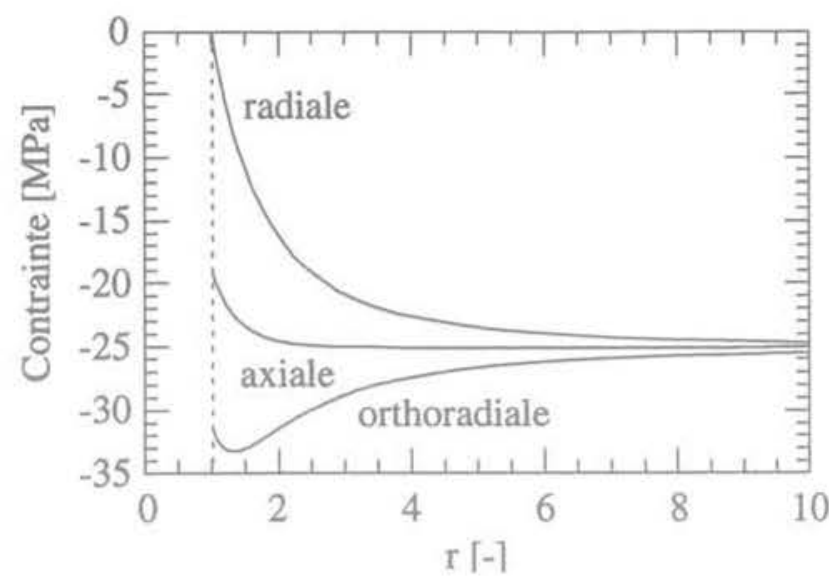

คG. 20 Répartition des contraintes autour de la galerie circulaire dans un massif élastoplastique.

Stress state around a circular cavity in elastoplastic medium.

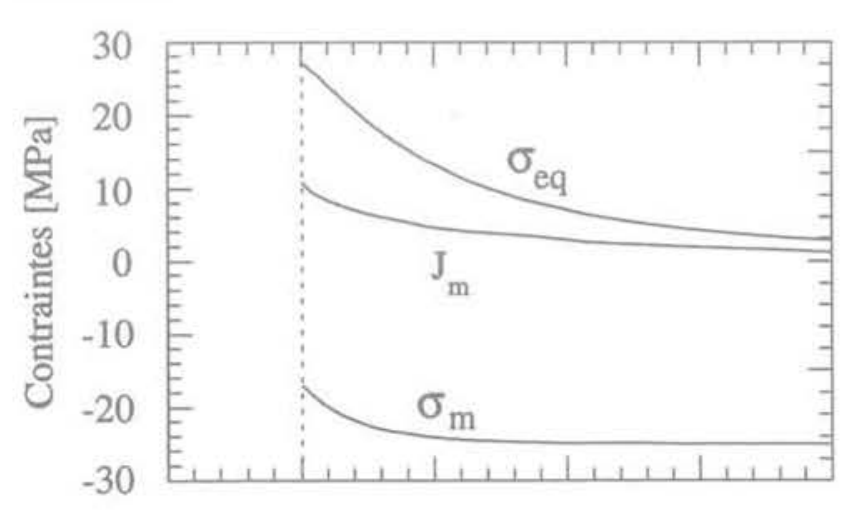

FG. 21 Évolutions des invariants des contraintes de Cauchy autour de la cavité cylindrique dans un massif élastoplastique.

Evolution of stress invariants around a cylindrical cavity in elastoplastic medium.

limite située à deux rayons. Notons que le critère de plasticité est atteint en permanence.

On se rapproche en paroi du critère de rupture sans que celui-ci ne soit véritablement atteint.

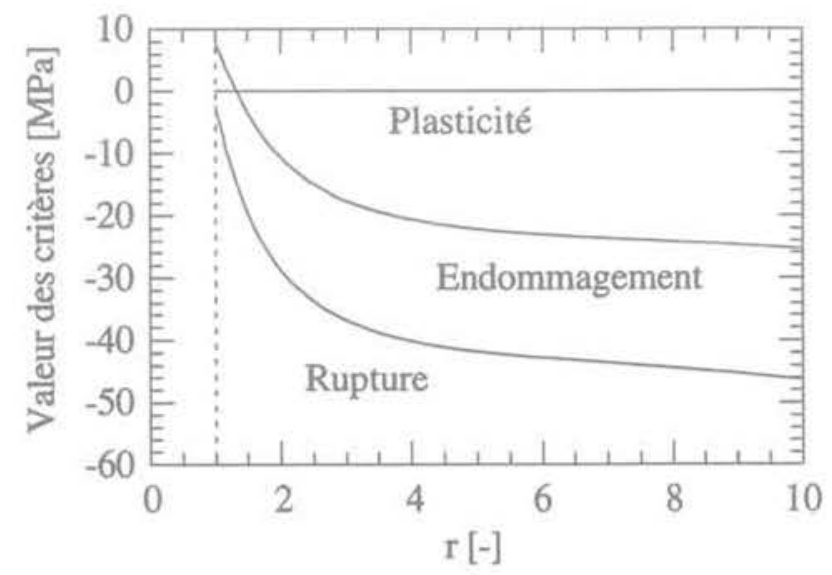

FiG. 22 État de contrainte par rapport aux critères dans le cas d'une cavité cylindrique dans un massif élastoplastique.

Stress state with respect to the criteria, in case of cylindrical cavity in elastoplastic medium. 
La figure 23 permet d'observer que la plastification du massif exprimée par la variable d'écrouissage, et notamment l'endommagement au voisinage de la galerie traduit par la dilatance sont relativement modérés, les déformations plastiques déviatoriques et sphériques étant d'environ $10^{-3}$ et $10^{-4}$ respectivement, tandis que la convergence de la cavité (déformation orthoradiale) calculée en élastoplasticité est de $8.10^{-3}$, la convergence élastique étant de $2.10^{-3}$.

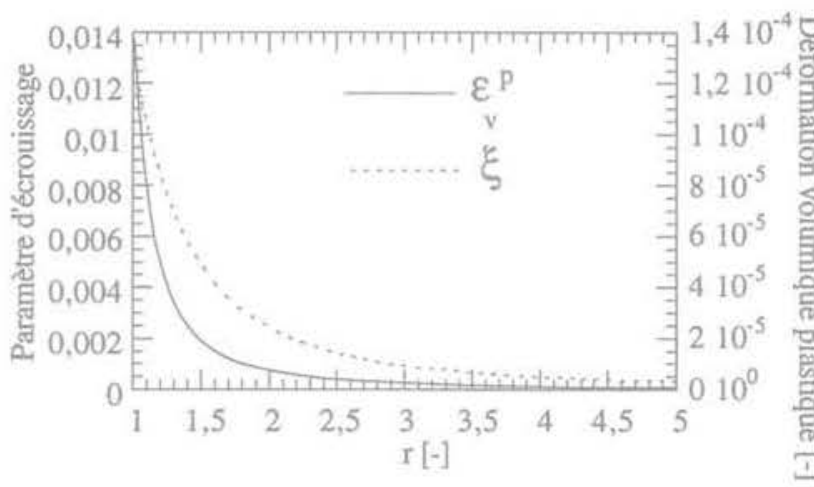

FiG.93 Évolution du paramètre d'écrouissage et de la déformation volumique irréversible autour de la galerie.

Strain hardening parameter and volumetric plastic strain around a cylindrical cavity in elastoplastic medium.
Aux profondeurs comprises entre $250 \mathrm{~m}$ et $1 \mathrm{~km}$, où se trouvent nombre d'ouvrages en milieu salifère, ce modèle montre que le voisinage des structures souterraines peut être plastifié et endommagé. Cela peut remettre en cause l'étanchéité hydraulique du sel visà-vis d'un stockage souterrain, même si cet endommagement n'entraine pas souvent la rupture de l'ouvrage.

\section{5}

\section{Conclusion}

Le comportement à court terme du sel gemme a été étudié en tenant compte de l'endommagement et en s'appuyant sur des travaux antérieurs ainsi que sur nos travaux expérimentaux. En suivant des chemins de contrainte en compression et en extension, un modèle de comportement élastoplastique non standard, généralisé à trois dimensions, a été proposé pour rendre compte de l'endommagement du sel gemme. Ce modèle permet d'évaluer la plastification et l'endommagement des structures souterraines dans le sel gemme. Bien que conçus et mis en œuvre en particulier pour le sel gemme, la méthode et le principe du modèle présentés sont susceptibles d'être utilisés pour d'autres roches ductiles.

\section{Remerciements}

Les auteurs tiennent à remercier l'Agence Nationale pour la Gestion des Déchets Radioactifs (ANDRA) pour le soutien actif qu'elle a apporté à la réalisation de cette recherche.

\section{$\overline{\text { Bibliographie }}$}

Aubertin M., Gill D.E., Ladanyi B. - * Lab. validation of a unified viscoplastic model for rocksalt 》, ISRM Aachen. 1991, p. 183-186.

Aubertin M., Gill D.E., Ladanyi B. is Modelling the transient inelastic flow of rocksalt n, 7 th Symp. Salt Tokyo, vol.L. 1993, p. 93-104.

Berest P. Brouard B. - « Behavior of sealed solution-mined caverns $n$, Solution Mining Research Institute Spring Meeting, New Orleans, Louisiana, 1995,26 p.

Cristescu N. - " Rock Rheology ». Kluwer Academic Publisher, 1989, 336 p.

Cristescu N.D. - « A general constitutive equation for transient and stationary creep of rock salt ग, Int. J. Rock Mech. Min. Sci \& Geomech., Abstr., vol. 30, $n^{\circ}$ 2. 1993, p. 125-140.

Davidge R.W., Pratt P.L. - « Plastic deformation and work hardening in $\mathrm{NaCl}{ }_{*}$, Phys. stat. Sol. 6, 1964, p. 759-776.

Habib P. - « La résistance au cisaillement des sols. \# Thèse de la faculté des sciences de l'université de Paris. La documentation technique du bâtiment et des travaux publics. Paris, $1953,40 \mathrm{p}$.

Hunsche U. - $\approx$ Fracture experiments on cubic rock salt samples. \# 1st Conf.
Mech. Behav. of Salt. Penns. State Univ. 1981 Nov. 9-11. Trans. Tech. Pub. 1984 p. $169-179$.

Hunsche U. - « Failure behav, of rock salt around undergr. cavities $x_{2}, 7$ th Symp. Salt, vol. I. Elsevier, 1993, p. 59-65.

Hunsche U. Albrecht H. $-\alpha$ Results of true triaxial strength tests on rock salt. $x$ Engng Fract. Mech., vol. 35, n² 4/5, 1990 p. $867-877$.

Laitai E.Z. Scott Duncan E.J. - « The mechanism of deformation and fracture in potash rock. ") Can. Geotech. J., 25. 1988, p. 262-278.

Lemaitre J., Chaboche J.-L. - « Aspect phénoménologique de la rupture par endommagement, n. J. Méc. Appl., vol. 2 , $n^{\circ} 3,1978$, p. $317-365$.

Luong M.P. - "Fondations sous chargement dynamique, cyclique et transitoire ${ }^{3}, 20^{\circ}$ anniversaire du Laboratoire de Mécanique des Solides de l'École polytechnique, 1981, p. $\mathrm{H} 1$ à H30.

Peach C.J. - is Deformation, dilatancy and permeability development in halite/anhydrite composites », Proc. 3rd conf. on the Mech. Behav, of Salt. sept., Palaiseau, France, 1993.

Pouya A. - « Approche micro-mécanique de la fissuration du sel gemme 3 , 7th ISRM Congr., Aachen, 1991, p. 319-322. Senseny P.E. Fossum A.F., Pfeife T.W. "Nonassociative constitutive laws for low porosity rocks. in Int. J. for Num. Anal. Meth. Geomech., vol. 7, 1983. p. 101-115.

Spiers C.J., Peach C.J. - « Development of dilatancy and permeability in rocks during creep : experiments on rocksalt as rock analog. p. Int. Geol. Congr., 198907-09, Washington D.C., vol. 3, 1989, p. $162-163$

Stokes R.J. - « Mech. properties of polycristaline NaCl. i Proc. British ceramic soc., vol. 8-6, 1966, p. 189-207.

Thorel - « Plasticité et endommagement des roches ductiles : application au sel gemme. n Thèse ENPC, 1994, 274 p.

Thorel L., Ghoreychi M. - $\alpha$ Rock salt damage. Exp. results and interpretation 1, Proc. 3rd Conf. Mech. Behav. Salt, Sept. Palaiseau. France, Trans. Tech. Pub. 1993, 15 p.

Van Sambeek L.L., Ratigan J.L., Hansen F.D. - « Dilatancy of rock salt in laboratory tests w. Int. J. Rock Mech. Min. Sci \& Geomech., Abstr., vol. 30, n 7, 1993. p. $735-738$. 